\title{
Ontological representation of tactile information for software development
}

\author{
Eirini V. Myrgioti *, Vasilios G. Chouvardas and Amalia N. Miliou \\ Informatics Department, Aristotle University of Thessaloniki, 54124 Thessaloniki, Greece \\ E-mails: \{emyrgiot, vchou, amiliou\}@csd.auth.gr
}

\begin{abstract}
The last years a growing body of research has been developed on tactile displays and interfaces, which can give physical form to digital information. In spite of the numerous tactile devices that have been constructed, the development of formal definition for tactile information still remains an open issue. In order for the software to better enhance the tactile information sensation, flow and perception and also drive tactile interfaces we need a formal representation of tactile information. The tactile information ontology proposed in this article analyzes the existing knowledge in tactile information domain, specifies the conceptual structure, and defines the processes that take part in the tactile information flow. Furthermore, the developed ontological representation encodes knowledge into computer understandable form and will allow the sharing and reuse of this knowledge by software tools. The proposed model will be used to identify how tactile displays can improve human-computer interaction, set the principles that are required to design effective user interfaces and assist the development of software for tactile displays. Along with the ontological model, a case study relative to the perception of roughness, generated by a tactile device, has been described.
\end{abstract}

Keywords: Tactile displays, tactile information, tactile interfaces, human-computer interaction, ontologies, software development

\section{Introduction}

Tactile displays are human-computer interfaces that utilize the sense of touch to present information. Tactile information refers to a set of signals that is created by a tactile display when different kind of stimuli are applied. These signals are conveyed to the skin receptors through the skin. The skin receptors transmit tactile signals to the nerve fibers which are responsible to convey the information through multiple pathways to the brain in order to form a perception of an object. The detection and extraction of the physical parameters of a touched object such as its shape, size, edges, texture, curvature and temperature is one of the main scopes of tactile interfaces in order to form a perception.

Scientific attention is directed to tangible interfaces that could be used in various applications enhancing perception of information in human-computer interaction. However, the design and development of tactile interfaces is not a trivial task, because software developers must have a thorough knowledge of the skin physiology and the nervous system structure and function, and a deep understanding of how the tactile information is conveyed. In order to develop useful tactile interfaces and effective software that would drive tactile displays we need a well-structured and formal representation of tactile information. Physiological and psychophysical knowledge would help us develop such a representation.

\footnotetext{
${ }^{*}$ Corresponding author: Eirini V. Myrgioti, Informatics Department, Aristotle University of Thessaloniki, 54124 Thessaloniki, Greece. Tel./Fax: +30 2310 991922; E-mail: emyrgiot@csd.auth.gr.
} 
Ontologies have gathered increased attention as they have been recognized to be useful not only in knowledge-based systems but also in the software development process (Devedzic, 2002; Rubin et al., 2007). An ontology defines the basic terms and relations comprising the vocabulary of a topic area in machine-interpretable way as well as the rules for combining terms and relations to define extensions to the vocabulary (Gomez-Perez, 2003). In Devedzic (2002); Gomez-Perez (2003), ontologies are used for communication between systems and people, design and development of knowledge-based systems and interoperable software components, and even classes of objects in object-oriented design. Furthermore, as acknowledged by Noy \& McGuiness (2001), the reasons that the ontology development is useful are: (1) to share common understanding of the structure of information among people, (2) to make domain assumptions explicit, (3) to enable reuse of the domain knowledge, (4) to analyze domain knowledge and (5) to separate domain knowledge from operational knowledge. The ontology captures the intrinsic conceptual structure of the domain. In order to build a knowledge representation language based on analysis, we need to associate terms with concepts and relations in the ontology and devise a syntax for encoding knowledge in terms of these concepts and these relations (Chandrasekaran et al., 1999).

Tactile information is a complex concept that contains many interrelated elements such as many kinds of stimuli, skin receptors (Iggo, 1984; Johansson, 1978; Macefield, 2005), nerves and brain areas (Bohlhalter et al., 2002; Hlushchuk \& Hari, 2006). Their properties and the relations between each element describe the procedure of generation and processing of tactile information and its flow from a tactile display to the brain. However, tactile information is modulated by the channel that is defined by the stimuli-receptors-nerves-brain-perception scheme and different features of a touched object are perceived following different pathways in the above mentioned scheme (Myrgioti et al., 2007). Modeling the existing knowledge of stimuli-receptors-nerves-brain-perception scheme provides a formal representation of the tactile information domain. Using formally specified domain knowledge in software design leads to a specific structure of the model (Hruby, 2005). However, we do not attempt to construct a model of touch considering all aspects of neurophysiology, but to model tactation in order to implement human-computer interaction through touch. Since we need a formal structure of tactile information, we will illustrate that constructing a tactile information ontology will assist software development for tactile displays.

As far as we know from the current bibliography, no other effort has been reported towards the construction of a tactile information ontology, for tactile software development. However, we currently witness an increasing number of bio-ontologies under development, primarily in the Open Biological Ontologies $(\mathrm{OBO})^{1}$ framework. The aim of this paper is twofold: (a) to form a formal tactile information definition and (b) to present a complete model of tactile information defined explicitly in terms of tactile information characteristics using ontologies that will assist the development of software for tactile displays. Furthermore, this model would be reusable by software agents for many applications.

This paper is organized as follows; Section 2 presents current research in the area and Section 3 introduces the elements that take part in the tactile information flow, i.e., the receptors, the nerves and the brain. Section 4 presents the design concepts and the methodology that have been followed in the development of the ontology, Section 5 presents the tactile information ontology along with diagrams that represent the class hierarchy and complete the ontology description. Section 6 presents the ontological relations and in Section 7 a case study is presented providing a validation to our model. Finally, in Section 8 conclusions and further research directions are discussed.

\footnotetext{
${ }^{1}$ http://obo.sourceforge.net.
} 


\section{Related work}

Tactile and haptic interaction offers an independent sensory channel that the brain can process to further enhance a user's experience in a multimodal environment. Haptic interaction relates to all aspects of touch and body movement and their application to computer interaction (Hale \& Stanney, 2004). This involves not only sensation and perception, but also motor and cognitive aspects of active movement for which detailed motor plans are created, stored in memory, and compared to feedback from receptors located in the muscles, joints, and skin. Although there is a lot of research in the area of tactile sensation (Bolanowski et al., 1988; Hale \& Stanney, 2004; Iggo, 1984; Macefield, 2005), and tactile information processing (Bohlhalter et al., 2002; Gardner \& Kandel, 1991; Hlushchuk \& Hari, 2006), which play an important role in the design and development of tactile interfaces, a formal knowledge representation of tactile information has not yet been developed.

Ontology is a specification of an abstract, simplified view of the world that we wish to represent for some purpose (Gruber, 1993). Therefore, ontology defines a set of representational terms that are called concepts. Relationships among these concepts describe the target world (Khan \& Luo, 2002). Research work in ontology design has produced a variety of different ontology classifications, from ontologies that represent general world knowledge, domain-specific ontologies, and knowledge representation systems that embody ontological frameworks (Noy \& Hafner, 1997).

Important research work in object-oriented modeling has already been developed based on ontologies (Evermann \& Wand, 2005; Shegogue \& Zheng, 2005). In object-oriented paradigm, domain ontology defines the metamodel for application models in this domain. In Shegogue \& Zheng (2005) has been demonstrated that the utility of ontology terms can be enhanced by object-oriented technology, and ontology terms can be integrated into an object-oriented model serving as a basis for the generation of object functions and attributes. By applying object-oriented methodologies and concepts the various domains of a specific ontology can be coordinated into one model. Specifically, ontology terms were presented in a parent-child hierarchy connected by "is-a" (generalizations) and "part-of" (composition) relationships. Read from top to bottom, the ontology terms proceed from more specific to less specific (Shegogue \& Zheng, 2005). Furthermore, in Evermann \& Wand (2005), ontologies have been used to show how basic ontological concepts can be mapped onto an object-oriented equivalent, indicating that object-oriented languages are expressive enough to model real-world application domains. In Bonacin et al. (2004), heuristics and rules have been developed in order to design class diagrams based on ontology charts. By applying them it is possible to produce a first draft of class diagrams useful in a system implementation directed to object-oriented programming paradigm.

Some software engineering researchers view ontologies as a "vocabulary" for a specific domain representing conceptual elements and the relationships existing between them. Though, the ontology is not the vocabulary itself, but what the vocabulary represents, since the translation of this vocabulary into another language will not change the ontology (Chandrasekaran et al., 1999). However, many researchers have concluded that ontologies of software systems application domain, or of its design and construction processes, are of great assistance in avoiding problems and errors at all stages of the software product life cycle: from the initial requirements analysis, through the development and construction phase, and finally with the maintenance stage (assuring greater understanding of the modification requests, better understanding of the maintained system, etc.) (Ruiz \& Hilera, 1998). Additionally, numerous authors have perceived ontologies from different views. Our developed ontology according to generality level is classified in the domain ontologies and captures the knowledge valid for the particular type of domain. In accordance with the type of conceptualization structure (van Heijst et al., 1997), our ontology 
belongs to knowledge representation ontologies that captures the representation primitives used to formalize knowledge in knowledge representation paradigms. Based on the level of richness of its internal structure the developed ontology belongs to frame ontologies including such class as properties, which can be inherited by other classes in lower levels of a formal "is-a" taxonomy. Finally, depending on the subject of conceptualization, our developed ontology falls into application ontologies that contain the necessary knowledge for modeling a particular domain (Gomez-Perez, 2003).

\section{Tactile information flow}

The representation of tactile information, in terms of "receptors-nerves-brain" (Fig. 1), sets the foundations for analyzing tactile information. In the following section, we describe the stages of tactile information flow and their role in tactile information processing.

\subsection{Receptors}

The skin is considered as the layer receiving external stimuli and transmitting energy to the receptors. The characteristics of the skin vary among different human groups (blind people have thinner skin at the fingertips), and parts of the body. However, the major part of current research work is related to the glabrous skin (without hair), which is most effective for detailed tactile information and most of the applications developed so far are used on the finger. The skin is sensitive to pressure, vibration, temperature and current.

The skin's complexity and function, are factors that must be considered in the design of tactile interfaces. The skin contains different kind of receptors that are arranged in different depth layers. Receptors analyze and deconstruct stimulus information. Specifically, receptors in the skin transduce the physical energy of a tactile stimulus into electrical signals. The receptors that are related to tactile information are organized in four categories that are: mechanoreceptors, proprioceptors, thermoreceptors and nociceptors (Iggo, 1984).

\subsubsection{Mechanoreceptors}

They are arranged in different depth layers and are classified into four categories; rapidly adapting I and II (RA-I and RA-II) and slowly adapting I and II (SA-I and SA-II), whose end organs are Meissner corpuscles, Pacinian corpuscles, Merkel cells and Ruffini endings, respectively (Iggo, 1984; Bolanowski et al., 1988; Johansson, 1978; Macefield, 2005; Pasterkamp, 1999). Rapidly adapting mechanoreceptors respond only at the beginning and end of a stimulus while slowly adapting respond throughout the stimulus. The sensitivity of mechanoreceptors in a received signal depends on the receptors properties (Hale \& Stanney, 2004). Table 1 outlines the categories of the mechanoreceptors and their respective properties. The analysis of each property leads to different levels of appropriateness for the construction of a tactile interface.

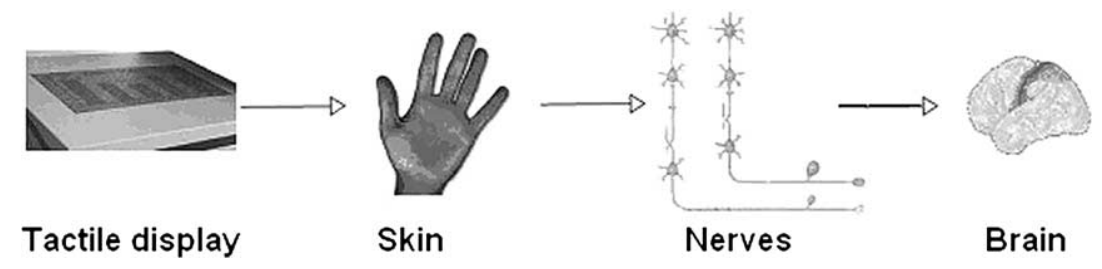

Fig. 1. Tactile information flow. 
Table 1

Human mechanoreceptors and their characteristics

\begin{tabular}{|c|c|c|c|c|}
\hline Properties & $\begin{array}{l}\text { Meissner } \\
\text { corpuscles }\end{array}$ & $\begin{array}{l}\text { Merkel } \\
\text { cells }\end{array}$ & $\begin{array}{l}\text { Ruffini } \\
\text { endings }\end{array}$ & $\begin{array}{l}\text { Pacinian } \\
\text { corpuscles }\end{array}$ \\
\hline Modalities & $\begin{array}{l}\text { Stroking, } \\
\text { fluttering }\end{array}$ & $\begin{array}{c}\text { Local stress-strain } \\
\text { (pressure) }\end{array}$ & Skin stretch & Vibration \\
\hline Frequency range (most sensitive at) & $\begin{array}{l}10-300 \mathrm{~Hz} \\
(20-50 \mathrm{~Hz})\end{array}$ & $\begin{array}{l}0.4-100 \mathrm{~Hz} \\
\quad(7 \mathrm{~Hz})\end{array}$ & $7 \mathrm{~Hz}$ & $\begin{array}{c}40-800 \mathrm{~Hz} \\
(200-300 \mathrm{~Hz})\end{array}$ \\
\hline Spatial resolution & $2-8 \mathrm{~mm}$ & $0.5 \mathrm{~mm}$ & $1 \mathrm{~cm}$ & $2 \mathrm{~cm}$ \\
\hline Receptive field & Small & Small & Large & Large \\
\hline Adaptation & Rapid (RA-I) & Slow (SA-I) & Slow (SA-II) & Rapid (RA-II) \\
\hline Receptors $/ \mathrm{cm}^{2}$ (at the fingertip) & 140 & 70 & 9 & 21 \\
\hline Physical features to be sensed & $\begin{array}{l}\text { Shape, } \\
\text { edges }\end{array}$ & $\begin{array}{l}\text { Curvature, } \\
\text { roughness }\end{array}$ & $\begin{array}{l}\text { Skin stretch, } \\
\text { motion direction }\end{array}$ & $\begin{array}{l}\text { Texture, } \\
\text { size }\end{array}$ \\
\hline
\end{tabular}

Meissner corpuscles: They are the shallowest mechanoreceptors located below the surface by about $0.7 \mathrm{~mm}$. They provide the most precise localization of touch, as they have the smallest receptive fields (2-8 mm diameter) (Pasterkamp, 1999), they are most sensitive in midrange stimulation frequency $(20-50 \mathrm{~Hz})$ and they mediate sensations of stroking and fluttering (Gardner et al., 1991; Macefield, 2005).

Merkel cells: They are most responsive to extremely low frequencies $(0.4-100 \mathrm{~Hz})$ and they have small receptive fields. Merkel cells are sensitive to physical deformation produced by local stress (pressure) and local strain of the skin (Gardner et al., 1991). Selective activation of Merkel cells produce sensations of steady pressure to the skin (Bolanowski et al., 1988). Only the Merkel cells population provides a neural image of the stimulus and its orientation that could count for the psychophysical behavior (Johnson, 2001).

Ruffini endings: These receptors are most sensitive to skin stretch. The Ruffini endings play an important role firstly in the perception of the direction of object motion or force when the motion or direction of force produces skin stretch and secondly, along with the muscle spindles and joint afferents, in the perception of shape at the hand and finger position through the pattern of skin stretch produced at each hand (Johnson, 2001).

Pacinian corpuscles: They are the deepest and most sensitive mechanoreceptors and comprise about 40 percent of all mechanoreceptors in the glabrous skin of the human hand (Pasterkamp, 1999). They are located below the surface by about $2 \mathrm{~mm}$ and they can be activated at high frequencies (40$800 \mathrm{~Hz}$ ) with a maximum sensitivity near $300 \mathrm{~Hz}$ (Bolanowski et al., 1988). Pacinian corpuscles are responsive to changes of frequency and area whereas the rest of the mechanoreceptors appear to be most responsive to changes in gradients on the surface of the skin (Verrillo, 1985). Selective activation of Pacinian corpuscles produces sensations of vibration but not of steady pressure (Gardner et al., 1991).

The properties of slowly and rapidly adapting mechanoreceptors define their sensitivity in the received signals (Gescheider et al., 2004; Hale \& Stanney, 2004) and must be considered in the construction of a tactile display and the design of tactile interfaces. These are:

- The density of the receptors differs among the different parts of the body. A large number of receptors in a given area result in high spatial acuity. 
- The frequency range of signals received by the receptor indicate the frequencies by which the receptor is stimulated.

- The receptors' receptive field defines the region of the skin through which a tactile stimulus is conducted to reach the nerves and differs in size and response between the different kind of receptors.

- The spatial resolution is the ability to distinguish a pair of nearby stimuli and varies between receptors classes due to differences in receptive field size.

- The time range is defined as the response of the receptor and is determined by how quickly the energy is received and lost by the receptor (Hale \& Stanney, 2004).

\subsubsection{Proprioceptors}

Proprioception is the sense of position and movement of limbs and body without using vision. There are two submodalities of proprioception: (a) the sense of stationary position of the limbs (limb-position sense) and (b) the sense of limb movement (kinesthesia). Three types of receptors in muscle and joints signal the stationary position of the limb and the speed and direction of limb movement:

1. specialized stretch receptors in muscle, termed muscle spindle receptors (Pearson \& Gordon, 1991),

2. Golgi tendon organs, receptors in the tendon that sense static and active limb positioning (Hale \& Stanney, 2004) and

3. receptors located in joint capsules that sense flexion or extension of the joint (Gardner et al., 1991; Macefield, 2005).

In addition, stretch-sensitive receptors in the skin (Ruffini endings) also signal postural information.

\subsubsection{Thermoreceptors}

They are activated when they sense changes in skin temperature. They contribute: (a) to the total sensory information about an object making contact with the skin and (b) in accessing body heat loss or gain that occurs over a large part of the body surface (Darian-Smith \& Johnson, 1977). Humans recognize four distinct types of thermal sensations: cold, cool, warm and hot. These thermal sensations result from temperature differences between the skin (normal temperature $34^{\circ} \mathrm{C}$ ) and the objects contacting the body. Although it seems that the skin is sensitive to temperature, our receptors cannot measure the exact temperature of the surface but, they rather feel the thermal energy flow (Gescheider et al., 1997).

Warm receptors (warm-hot sensation) are active at steady temperatures of $29-45^{\circ} \mathrm{C}$ while they are most sensitive at $45^{\circ} \mathrm{C}$ (Gardner et al., 1991). Cold receptors (cold-cool sensation) differ from warm receptors in terms of the steady-state temperature range to which they respond and in their peak temperature sensitivities. They respond to steady-state temperatures of $5-40^{\circ} \mathrm{C}$ while they are most active at $25^{\circ} \mathrm{C}$. When skin temperature rises over $45^{\circ} \mathrm{C}$ or falls below $15^{\circ} \mathrm{C}$, receptors that sense the pain (nociceptors) are stimulated and humans feel pain rather than warm or cold sensation respectively (DarianSmith \& Johnson, 1977).

\subsubsection{Nociceptors}

They are responsible for the pain signals that can damage the skin tissue. They are distinguished in: mechanical, that are excited by sharp objects that penetrate, squeeze, or pinch the skin, thermal which respond to extremes of temperature as well as strong mechanical stimuli, and polymodal which respond to a variety of destructive mechanical, thermal, and chemical stimuli (Gardner et al., 1991; Iggo, 1984). 


\subsection{Nerves}

The skin receptors are innervated by different kinds of nerve fibers (neurons). The nerve fibers are classified according to their size (diameter) and the conduction velocity of the signals. Mechanoreceptors and proprioceptors are innervated by neurons with large diameter that conduct the signals from the skin rapidly. Thermoreceptors and nociceptors are innervated by neurons with small diameter that conduct the signals more slowly (Gardner et al., 1991). Touch, proprioception, temperature and pain are conveyed in two separate ascending pathways to the brain thalamus and cerebral cortex. The first pathway carries information about tactile sensation and limb kinesthesis while the second pathway carries information about temperature and pain. In the case of touch, primary sensory neurons with receptors at the skin translate stimulus energy into neural events that initiate activity in precise pathways that include several processing stages before they terminate in the somatosensory areas of the cerebral cortex of the brain. Nerves play a significant role in tactile information flow because they are the means of conveying the tactile signals from the receptors at the skin to the brain. Additionally, understanding their role would provide important information on how quickly a user of a tactile device will perceive the information exerted and this fact will lead to a better design of tactile interfaces.

The types of nerve fibers that are related to cutaneous sensation are:

- The I type which consists of Ia nerve fibers that mediate muscle stretch from muscle spindles, and Ib nerve fibers that mediate limb and joint movement from Golgi tendons and Golgi endings.

- The II type mediate muscle stretch from muscle spindles as Ia fibers.

- The A type consists of $A \beta$ fibers and $A \delta$ fibers. A $\beta$ fibers mediate vibration, pressure, skin stretch, fluttering and stroking from mechanoreceptors. A $\delta$ fibers conduct signals from cold receptors as well as primary pain sensation.

- C fibers are responsible for conveying signals of warm receptors and secondary pain sensation (Vallbo et al., 1979).

\subsection{Brain}

A large population of nerve fibers convey information from the skin receptors to the brain which is able to integrate spatial, temporal, kinesthetic and other information about tactile stimuli in order to create perception of an object (Recanzone et al., 1992). Sensory information is processed in a series of relay regions within the brain. Each of the regions has essentially the same map about the parts of the body and provide information about different types of sensations. Any point on the skin is represented in the cortex by a population of cortical cells connected to the afferent fibers that innervate that point on the skin. When a point on the skin is touched, the population of cortical neurons connected to the receptors at that location is excited. Stimulation of another point on the skin activates another population of cortical neurons. We perceive contact at a particular location on the skin because a specific population of neurons in the brain is activated. The somatic sensory cortex has three major sections that are responsible for the tactile sensation: the primary (S-I) and secondary (S-II) somatosensory cortices and the posterior parietal cortex. Each of them contains areas that are responsible for processing different signals transmitted by the skin, as analyzed below.

\subsubsection{Primary somatic cortex (S-I)}

The primary somatic cortex (S-I) (Gardner \& Kandel, 1991) contains four areas, commonly referred to as: area 1, area 2, area $3 a$ and area $3 b$. Although each of the areas has essentially the same body 
map, it receives information from different receptors and it represents different types of information. Basic processing of tactile signals takes place in area 3, while more complex or higher-order processing occurs in area 1 and area 2 . Specifically, area 1 receives input from rapidly adapting (RA) receptors and senses the object's size, shape and texture. Area 2 receives information for pressure and for joint position (Ruffini endings and Golgi tendon organs) and both tactile information and proprioceptive information are combined to mediate the tactile recognition of objects (Bohlhalter et al., 2002). Neurons in area 2 sense complex features, such as the direction of motion across the hand, the curvature of surfaces, the orientation of edges, or the spacing of ridges on textured surfaces. Area $3 a$ receives input from muscle stretch receptors (muscle spindles) while area $3 b$ receives input from slowly adapting receptors. Finally, the S-I cortex sends outputs to the posterior parietal cortex, where integration with other senses and the opposite limb occurs and where an overall picture of the body is formed.

\subsubsection{Secondary somatic cortex (S-II)}

The secondary somatic sensory cortex (S-II), is innervated by neurons from each of the four areas of S-I. S-II also innervates regions of the temporal lobe believed to be important for tactile memory. S-II neurons have larger receptive fields and show more complex responses that are not consistently related to the attributes of the stimulus (Klatzky \& Lederman, 2002).

\subsubsection{Posterior parietal cortex}

Important somatosensory cortical areas are also located in the posterior parietal cortex (Gardner \& Kandel, 1991). Area 5 integrates tactile signals from proprioceptive inputs, from the underlying muscles and joints and encodes the shape of an object. Area 7 receives visual as well as tactile and proprioceptive inputs allowing integration of stereognostic and visual information. The posterior parietal cortex plays an important role in sensory initiation and guidance of movement. Neurons in these areas are involved in the later stages of processing, have more complex feature-detecting properties, receive convergent input from several submodalities, and have larger receptive fields than first-order cortical neurons.

In (Fig. 2), a diagram of how tactile information is conveyed through the tactile channel, is represented. Tactile features of an object are transmitted to tactile display actuators causing direct actuator stimulation. The channel of transmitting the tactile signals to the receptors is the skin making use of its mechanical or electrical properties. The receptors transmit the tactile signals that receive to the cortex of the brain through the nerve channel. The processing of tactile signals by the brain leads to the action of perception of the touched object.

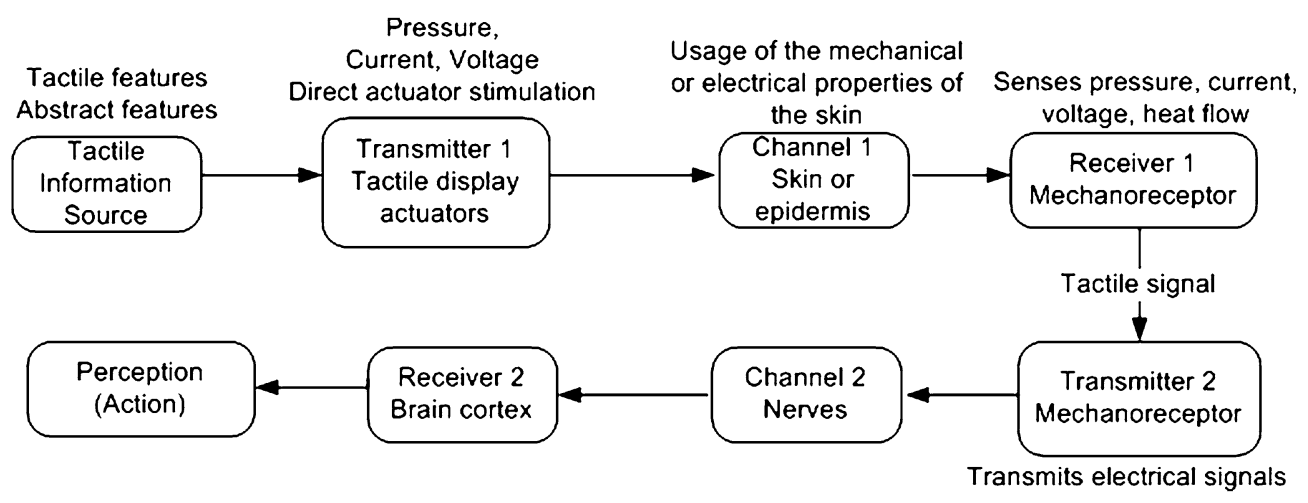

Fig. 2. Tactile information flow diagram. 


\section{Design considerations}

In order to write useful application software we need a model of the relevant world (entities, properties and relations). Object-oriented design of software systems depends on an appropriate domain ontology. Specifically, the result of object-oriented analysis is a draft of the domain ontology relevant to the application (Devedzic, 2002). Objects, their attributes and their procedures are more or less mirror aspects of the domain that is relevant to the application (Chandrasekaran et al., 1999). Furthermore, ontologies are especially useful for the development of reusable, high-quality software, as they provide unambiguous terminology which can be shared by all the development processes. Thanks to ontologies, the eliciting and modeling of the requirements phase can be carried out in two steps: in the first, general knowledge of the domain is obtained and specified in one or more ontologies. In the second step the obtained ontologies are used as inputs to develop the specified applications. The constructed ontology also serves as the basic vocabulary to speak about the domain and is a base for the development of the specific conceptualizations for the applications that are going to be constructed (Ruiz \& Hilera, 1998).

The application of ontologies to support the design and development of systems, specifically software, may have many objectives (Ruiz \& Hilera, 1998).

1. The role that ontologies play in specifications depends on the level of formality and automization within the methodology of the system design. From an informal perspective, ontologies assist in the requirements identification process and in the understanding of the relationships between components.

2. The formal ontologies allow for the semi-automized consistency check of a software system with respect to the declarative specifications that the ontology presumes.

3. By characterizing the domain classes and tasks within the subdomains, the ontologies could provide a framework to determine the aspects of the ontology that can be reused between different domains and tasks.

4. An ontology can be used as metadata, serving as an index for a repository of information.

5. The consistency checking may be automatic if a formal representation of knowledge exists.

6. Using ontologies allows an improvement of the documentation and a reduction in maintenance costs which is one of the main efforts made during the software system's maintenance phase.

7. In the process of building knowledge-based systems, speed and reliability may be increased when an existing ontology is used as a starting point and guide for the knowledge acquisition.

The ontology developed in our work includes the basic entities of the tactile information domain and their relations which are interpreted in a declarative way. For the design of the tactile information ontology, the steps of the tactile information flow are followed. Different kind of stimuli applied to a tactile display, produce information that is conveyed to the skin through its receptors. Next stage includes the transmission of information to the brain through nerves in order to integrate perception. For the development of the tactile information ontology we consider the different entities such as the types of skin receptors, the types of nerve fibers, the brain and its areas, the perceptive features of a touched object and also the different processes such as the stimulation of the receptors, the tactile signal transmission by receptors, by nerves and the perception. Moreover, we take into account the properties and the relations among the used concepts.

An ontology development can be expressed with different formalisms such as the Knowledge Interchange Format (KIF) (Gruber, 1993), the Web Ontology Language (OWL), the Unified Modeling Language (UML) (Kogut et al., 2002), or other ontology representation languages (Uschold \& King, 1995) 
and (Cranefield \& Purvis, 1999). UML is a formalism that is generally accepted for object-oriented design but it has some restrictions in the expressiveness of the ontological models. However, in cases where an application is to be written in an object-oriented framework, it is natural to generate a UML model from an existing ontological model (Mika, 2007).

\subsection{Methodology}

To normalize a domain ontology, it is important to find (i) an appropriate set of trees that form its skeleton and that represent ontologically significant categorial distinctions, and (ii) an appropriate set of binary relations. Fully structuring domain ontologies in a way that makes them computer-tractable and interoperable, as well as in a way that renders the information that they contain as clear, rigorous, and unambiguous as possible, requires the use of formal or "upper-level" ontologies. In addition, the important issues of terminology selection, term-definition, and classification in the sense of the construction of tree-like structures organizing the information contained in a domain ontology all benefit from and can be better understood in the context of, an explicitly defined formal ontology (Spear, 2006). The role of an upper-level ontology is to provide the basic categories within which different tree structures reside, and also to provide a list of binary relations together with axioms that specify their semantics (Bittner $\&$ Barry, 2004). There are currently multiple upper-level ontologies such as DOLCE ${ }^{2}$ (Gangemi et al., 2002), (Masolo et al., 2003), SUMO (Suggested Upper Merged Ontology) ${ }^{3}$ (Niles \& Pease, 2001) and BFO (Basic Formal Ontology) ${ }^{4}$ (Grenon et al., 2004). In the context of this paper we consider the BFO reference ontology because it is focused on the task of providing a genuine upper ontology which can be used in support of domain ontologies developed for scientific research, for example, in biomedicine, even though BFO does not contain physical, chemical, biological or other terms which would properly fall within the special sciences domains. Based on BFO we address (a) the basic categories that give raise to the different tree structures in tactile information domain and (b) the formal relations, which establish the relationships between classes within and across category trees.

The ontology presented in this work has been built using the Protege ontology editor, ${ }^{5}$ an environment where ontologies could be exported into a variety of formats including RDF, OWL and XML schema. Specifically, an OWL ontology may include descriptions of classes, properties and their instances. Given such an ontology, the OWL formal semantics specifies how to derive its logical consequences, i.e. facts not literally present in the ontology, but entailed by the semantics. Our ontology has been developed following the OWL semantics and its structure is based on BFO implementation with OWL. In this model, the ontology consists of a set of classes organized in the BFO hierarchy to represent the domain's concepts (substances and processes) and a set of properties associated to classes that describe their relations (Noy et al., 2001).

Our model addresses the entities and the relations between these entities in the tactile information domain. Specifically, our ontological analysis is based on the following statements: (i) classifying the entities of the tactile domain to continuants and occurrents, (ii) building the hierarchies of classes considering the current BFO taxonomy and following the top-down hierarchy approach (Uschold \& Gruninger, 1996), (iii) defining the relations between classes. We have also considered part of the subclass hierarchy

\footnotetext{
${ }^{2}$ http://www.loa-cnr.it/DOLCE.html

${ }^{3} \mathrm{http}: / /$ suo.ieee.org/.

${ }^{4}$ http://ontology.buffalo.edu/bfo/.

${ }^{5} \mathrm{http}: / /$ protege.stanford.edu/.
} 
tree of the SUMO ontology $y^{6}$ in relation to tactile perception. In SUMO, entities are divided into physical and abstract while physical entities can be objects or processes. The SUMO process hierarchy consists of classes defining biological and neurological processes including all aspects of perception along with tactile perception. The relations between classes, in the tactile information ontology, are established according to the foundational relations in the DOLCE (Masolo et al., 2004) and BFO (Grenon \& Smith, 2004) approaches and the relations used in the $\mathrm{OBO}^{7}$ framework.

\subsection{Basic formal ontology}

\subsubsection{Continuants and occurrents}

BFO (Basic Formal Ontology) is a "theory of the basic structures of reality" and has been developed at the Institute for Formal Ontology and Medical Information Science (IFOMIS). ${ }^{8}$ BFO is organized along two dimensions reflecting the opposition between different levels of granularity, from single molecules to whole populations of organisms, and the opposition between objects and processes (Grenon \& Smith, 2004). The central dichotomy among the perspectives represented in BFO concerns the modes of existence in time of the entities populating the world. There are entities that have continuous existence and a capacity to endure (persist self-identically) through time even when undergoing different sorts of changes and are called continuants or endurants. Continuants are said to be bound with respect to space and not with respect to time. This means that however we segment the interval of time during which a continuant exists, we find this continuant itself in every segment. BFO endorses in addition a view according to which the world contains occurrents, more familiarly referred to as processes, events, changes. Occurrents have, in addition to their spatial dimension, a temporal dimension, and they are bound with respect to time (in contradistinction to endurants) (Grenon et al., 2004). Processes characteristically have a beginning, a middle and an end. Some entities (planets, people, atoms) have an inherent ability to exist without support from other entities. Others require such support in order to exist: a viral infection is dependent upon a virus and upon the organism which is infected; the function of an organ is dependent upon the existence of the organ (Smith et al., 2003). Dependent entities include: qualities, states or conditions, functions and bodily features.

The architecture of the Basic Formal Ontology is based on the SNAP-SPAN theory (Grenon, 2003) which allows to talk not only about entities but also about the ontologies through which entities are apprehended. Entity refers to everything that exists or occurs in the spatiotemporal world. An ontology includes a taxonomy of the types of entities and relations that exist in a portion of the world seen from a given perspective. In BFO, each SNAP ontology represents the entities which fall under the categories of continuant entities and each SPAN ontology represents the entities which fall under the categories of occurrent entities. Both types of ontologies serve as basis for a series of sub-ontologies, each of which can be conceived as a window on a certain portion of reality at a given level of granularity.

\section{Tactile information ontology}

The tactile information ontology is a "theory" of a variety of entities and relations we find in the tactile interaction domain. The tactile information ontology is divided into two term hierarchies: the continuant hierarchy and the occurrent hierarchy.

\footnotetext{
${ }^{6}$ http://virtual.cvut.cz/kifb/en/toc/all.html.

${ }^{7} \mathrm{http}: / /$ obo.sourceforge.net.

${ }^{8} \mathrm{http}: / /$ www.ifomis.org/bfo.
} 


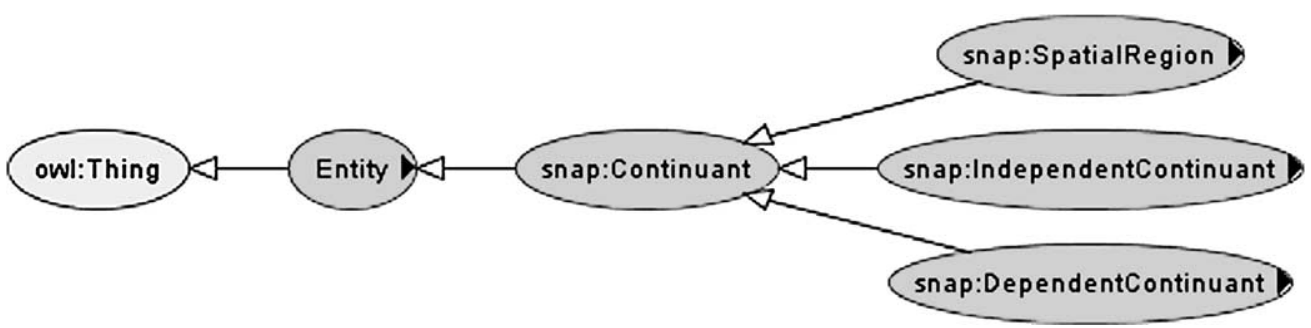

Fig. 3. The continuants subclasses.

\subsection{The SNAP tactile information ontology}

The continuants ontology is an inventory of all entities existing at a time in the tactile information domain. In Basic Formal Ontology, there are three distinct classes of continuants: the independent continuants, the dependent continuants and the spatial regions (Fig. 3). Continuant entities in the tactile domain are either parts of the human organism, such as brain, receptors, nerves, or they are located within the human organism (skin, palm, fingertip, muscles, joints) or they causally interact with the human organism in specific ways (users that interact with a tactile device, interaction points). The hierarchy of snap continuants classes is presented in Fig. 3.

\subsubsection{Independent continuants}

Independent continuants also referred to as substantial entities, have the following main features:

- they do not depend for their being sustained or maintained in existence upon other entities,

- they are enduring entities: they preserve their identity over time and through changes of various sorts,

- they have a location in space,

- they are self-connected wholes with physical boundaries which separate them off from other substances (Grenon et al., 2004).

Independent continuants are divided into: objects, aggregates of objects, object parts and sites:

Objects (Substances): Objects class contains independent continuants that their parts are not separated with each other with spatial gaps. Brain is an entity that falls in this category as a part of the human body and due to the fact that its identity is independent of that of other entities and can be maintained through time and through loss and gain of parts and qualities. Brain is enclosed by the cranial cavity. It receives information conveyed by the nerves and plays significant role in tactile perception and recognition of touched objects. Haptic device is also an objects class providing the means for a haptic interaction and haptic object is an objects class that contains all objects that can be explored and perceived through a haptic device.

Aggregates of objects: They are mereological sums comprehending separate substances as parts. In contradistinction to objects, aggregates have non-connected boundaries (Grenon et al., 2004). In the SNAP tactile information ontology, there are two subclasses of the class snap:AggregatesOfObjects: receptors and nerves. Receptors can be considered as aggregates of objects; they are a collection of four kind of receptors that are not connected with each other with physical boundaries and they are located in different parts of the body (Gardner \& Martin, 1991). Receptors class is divided into four subclasses according to the kinds of receptors that are related 
to the tactile information process and perception: mechanoreceptors, proprioceptors, thermoreceptors and nociceptors. Mechanoreceptors class contains the receptors that mediate the sense of touch and has two subclasses according to the receptors adaptation rate to a stimulus: rapidly adapting and slowly adapting. Rapidly adapting class is divided to $R A-I$ and $R A-I I$ subclasses and slowly adapting class is divided to $S A-I$ and $S A-I I$ subclasses. Proprioceptors class is classified according to the type of stimulation that activates the proprioceptors and their location, in three subclasses: Golgi Endings, Muscle spindles and Joint tendons. Thermoreceptors are divided into cold and warm receptors according to the temperature they perceive and nociceptors that respond to harmful stimulus have three subclasses: chemical, mechanical and polymodal. Nerves are a mereological sum of separate nerve fibers that differ from each other on morphology, function and location. There are four classes of nerves that are associated with the tactile information processing and these are: $I, I I, A$ and $C$ (Vallbo et al., 1979). Class I and class A have two subclasses: $I a$ and $I b$, and $A \beta$ and $A \delta$ respectively.

Object boundaries: Boundaries are those parts of objects that exist at the point where the object is separated off from the rest of the existing entities in the world and their dimension is lower than that of a substantial entity. In this SNAP subcategory belongs: the surface of the skin that is in contact with the tactile device and is divided into two subclasses: the palm and the fingertips. Other subclasses of the snap:ObjectBoundaries class are the object surface that refers to the boundaries of the touched object and the brain surface (cerebral cortex) that is the physical boundary between the brain cavity and the brain.

Fiat object parts: They are parts of an object but are not demarcated by physical discontinuities (Grenon et al., 2004). The regions of the brain cortex belong to this subcategory as they are parts of the brain which is an object and have no discontinuities in their structure. The regions of the brain cerebral cortex that are involved to the tactile perception and recognition of objects as mentioned above are: the primary somatic cortex, the secondary somatic cortex and the posterior cortex. Each of the regions is divided into areas according to their ability to receive, process and discriminate different signals. Primary somatic cortex has four subclasses: area 1, area 2, area $3 a$, area $3 b$ and posterior cortex is divided into area 5 and area 7.

Sites: They are entities that can be occupied by other continuants. A site is typically made of objects or parts of an object and the surrounding medium. In the tactile information ontology sites are: the cranial cavity which has a characteristic spatial shape and is occupied by brain regions and brain areas, the nerve canals occupied by nerve fibers, and joints, muscles and tendons occupied by receptors and especially proprioceptors. As described below, each site is associated at any given time with some specific spatial region (Grenon, 2003).

The hierarchy of independent continuants subclasses is presented in Fig. 4.

\subsubsection{Dependent continuants}

The class snap:DependentContinuant represents entities that endure in time and that inhere in or are born by other entities. This means that they depend on the existence of substantial entities. The hierarchy of dependent entities subclasses is shown at Fig. 5. Dependent entities class include the following subclasses:

- Qualities: In our work, we built two subclasses of the quality class regarding the tactile features of an object extracted by a haptic system, the material class and the geometry class. The material tactile features of an object are: hardness, temperature, texture and weight, and the geometry 


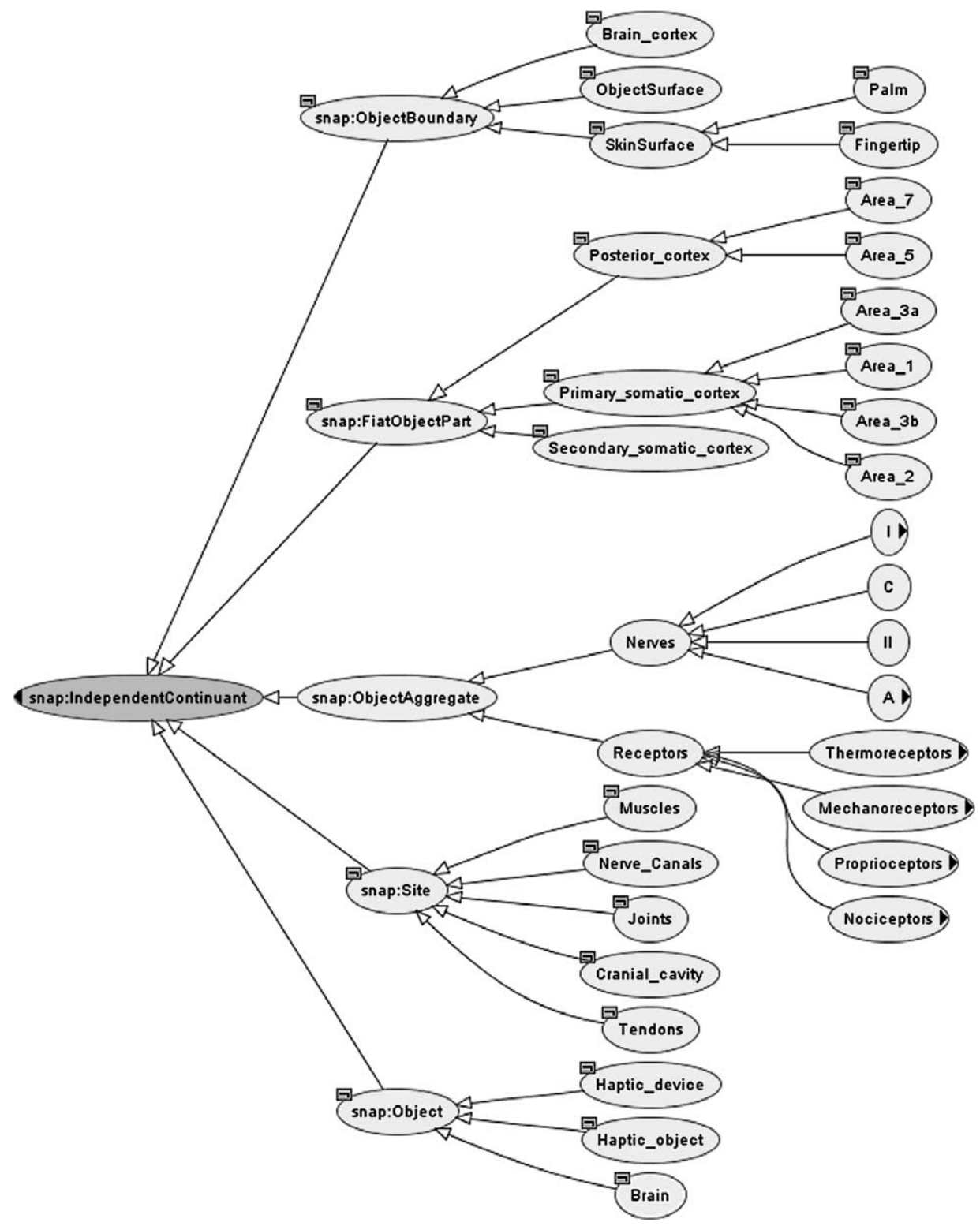

Fig. 4. The independent continuants hierarchy.

tactile features are: curvature, size and shape (Klatzky \& Lederman, 2002). Feature detection is a basic principle of cortical processing that allows the brain to find patterns common to stimuli of a particular class (Gardner \& Kandel, 1991).

- Roles: There are four subclasses of the role class: (i) the role of the different kind of stimuli to activate skin receptors, (ii) the role of the skin receptors to transmit information to the nerve fibers, (iii) the role of the nerves to drive tactile signals through different pathways and (iv) the role of the brain to integrate tactile perception.

- Functions: The subclasses of the functions class are: (i) the function of activation of the skin recep- 


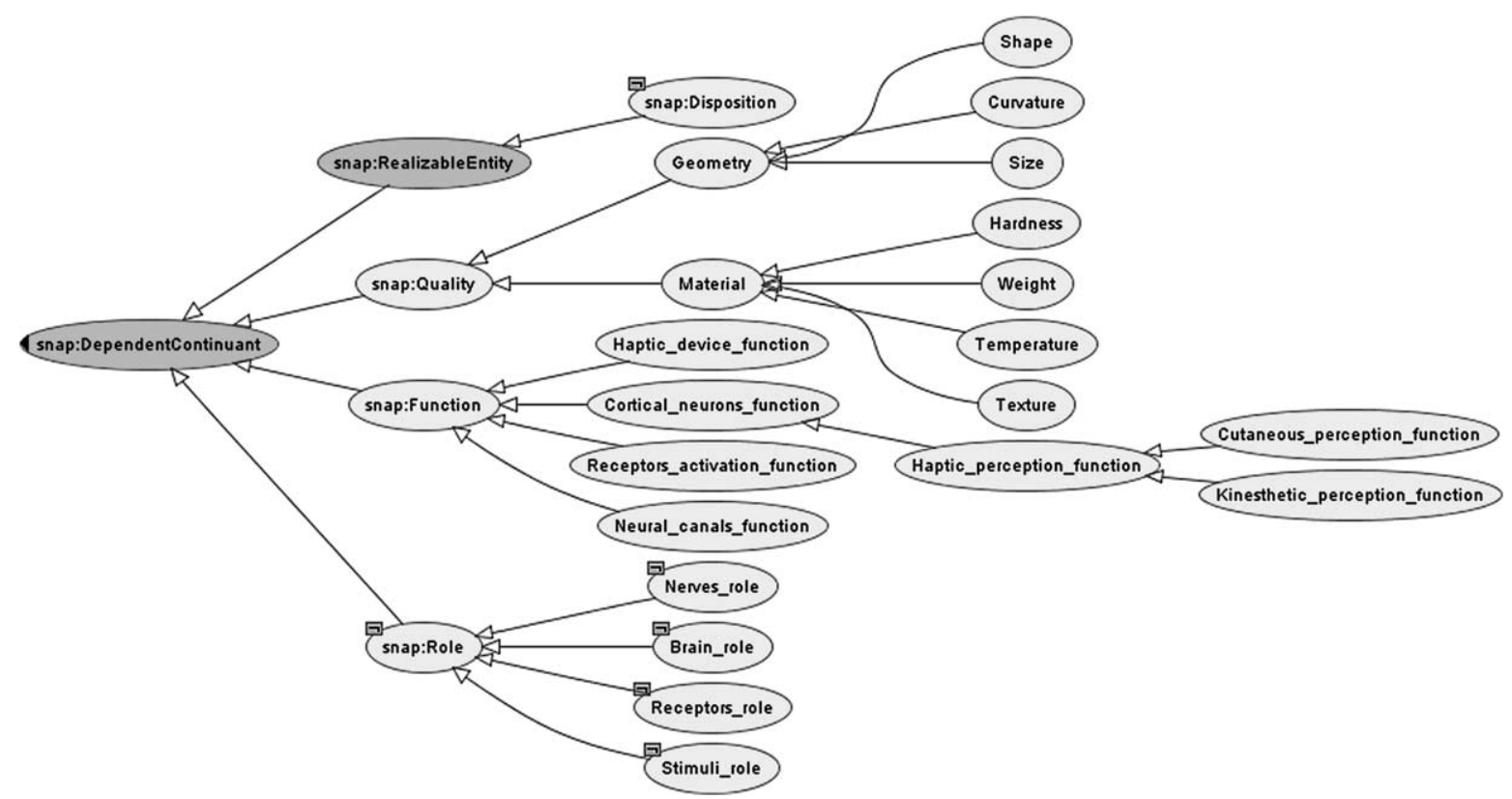

Fig. 5. The dependent continuants hierarchy.

tors when they receive a stimulus, (ii) the function of the nerve channels to enable tactile signals transmission, (iii) the function of a haptic device to create tactile information and (iv) the function of the cortical neurons to receive and process tactile signals and integrate haptic perception. The function of the cortical neurons to integrate perception can be divided into: the function to integrate cutaneous perception (processing tactile signals from the cutaneous receptors) and the function to integrate kinesthetic perception (processing tactile signals from the receptors located in muscles and joints). The function of the cortical neurons depends on the properties of each area which are:

- The receptive field differs among brain areas and becomes progressively more complex in areas that extract more features of a stimulus. Cortical receptive fields are larger than those of peripheral receptors due to convergence of inputs from simultaneously stimulated areas of the skin.

- Each brain area is activated by different stimulus indicating the dependence on the modality.

- Another property of the brain areas is the spatial resolution which allows humans to perform fine tactile discrimination of the touched object surface.

- The time response differs among brain areas (i.e., in areas 3a, 3b is $20 \mathrm{~ms}$ after touch) (Gardner \& Kandel, 1991).

\subsubsection{Spatial regions}

The class snap:SpatialRegions is the third of the main subclasses of the class snap:Continuant. An instance of the class snap:SpatialRegion is a spatial region, a part of space. All parts of space are spatial regions and only spatial regions are parts of space. All instances of the class snap:Continuant are spatial entities, that is, they enter in the relation of (spatial) location with spatial regions. As a particular case, the exact spatial location of a spatial region is the region itself. Space and spatial regions are entities in their own right existing independently of any entities which can be located in them. As we described above, in BFO the class snap:Site allows for a so-called relational view of space, that is to say, a view 


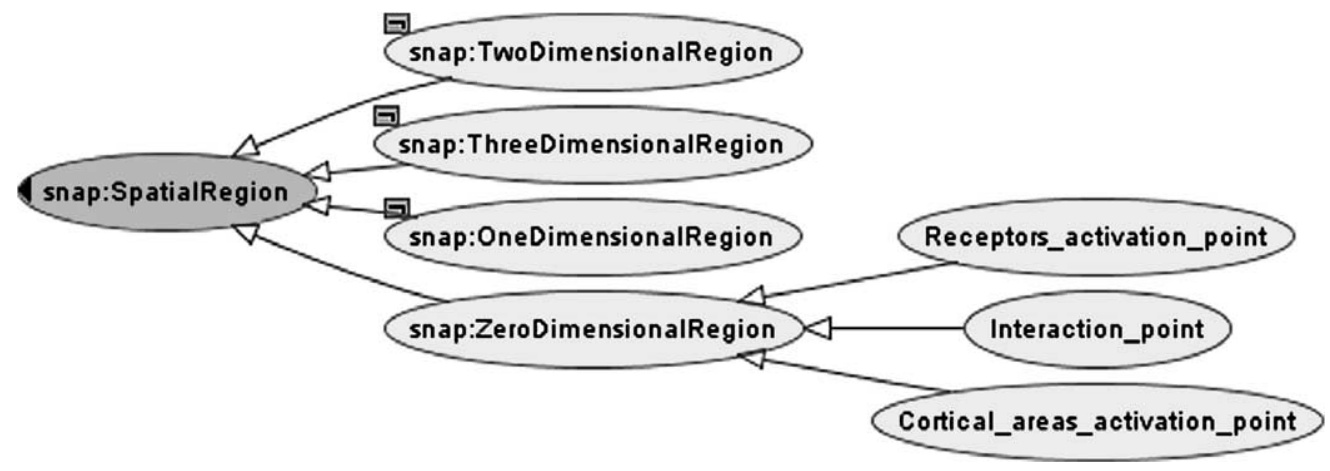

Fig. 6. The spatial regions hierarchy.

according to which spatiality is a matter of relative location between entities and not a matter of being tied to a space. The bridge between these two views is secured through the fact that while instances of the class snap:Site are not spatial regions, they are nevertheless spatial entities. The class snap:SpatialRegion has four subclasses: zero-dimensional, one-dimensional, two-dimensional and three-dimensional region. The zero-dimensional region class has three subclasses: interaction point class which includes the points where a haptic interaction occurs, the receptors activation point class which includes the points of the receptors that are activated and the cortical areas activation point class which includes the points of the brain areas that are activated by a stimulus. The hierarchy of spatial regions subclasses is shown in Fig. 6.

In Fig. 7 the complete hierarchy of the SNAP tactile ontology is presented.

\subsection{The SPAN tactile information ontology}

The SPAN tactile information ontology (Fig. 11) is a hierarchy of all the occurrents (processes and their temporal parts) that occur at the interaction with a tactile device. In BFO, the span:Occurents class has three main subclasses: Processual Entity, Temporal Region and Spatiotemporal Region (Fig. 8).

\subsubsection{Processual entities}

The class of processual entities stands to processes as the class of independent continuants (substantial entities) stands to substances. Processuals are occurents; they are entities which exist in time by occurring. They involve SNAP entities and they are dependent on these entities. The Processual entities class is divided into the following subclasses:

- Processes: This class contains entities that have beginnings and endings corresponding to real discontinuities in the processual order and involve no temporal gaps. As snap:objects occupy spatial regions, processes occupy spatiotemporal regions. In tactile information span ontology the subclasses of processes are: neurological system process and stimuli.

The neurological system process class, represents the process carried out by any of the organs or tissues of the neurological system (Smith et al., 2003). The perception of an object is the result of the combined processing of the tactile information by the brain areas. Haptic perception refers to tactual perception in which both the cutaneous sense and the kinesthetic sense convey significant information about objects (Loomis \& Lederman, 1986). The organization in terms of tactile, kinesthetic and haptic perception recalls the distinction between active and passive touch described by Loomis \& Lederman (1986); Klatzky \& Lederman (2002). They combined the presence or absence of motor 


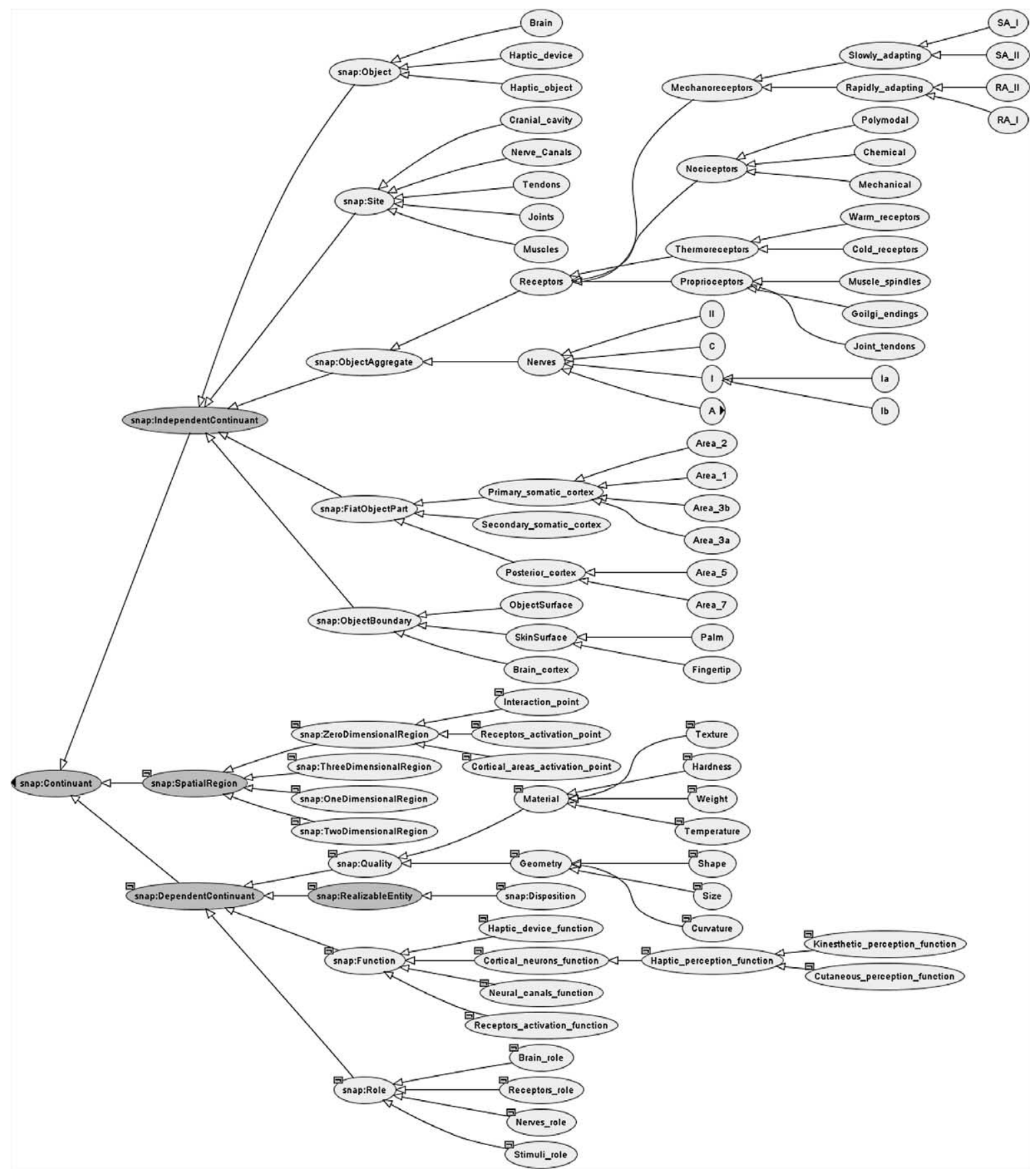

Fig. 7. The SNAP tactile information hierarchy.

control by a human over the pickup of information with the classification of sensory systems by the afferent inputs used (cutaneous, kinesthetic and haptic). This conjunction yielded five different "modes of touch": tactile (cutaneous) perception, passive kinesthetic perception (kinesthetic afferents respond without voluntary movement), passive haptic perception (cutaneous and kinesthetic 


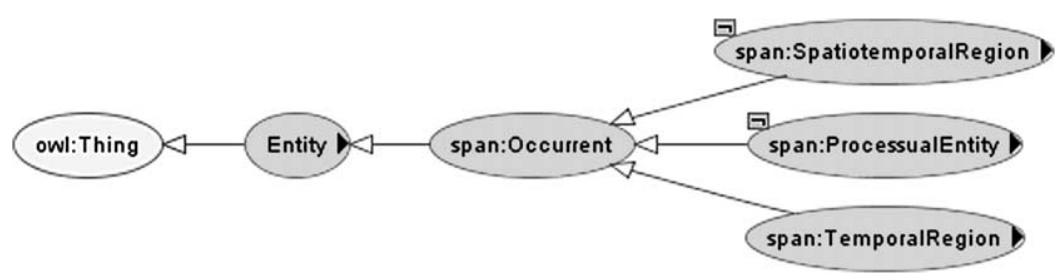

Fig. 8. The occurrents subclasses.

afferents respond without voluntary movement), active kinesthetic perception, and active haptic perception. The human only has motor control over the touch process in active kinesthetic perception and in active haptic perception (Klatzky \& Lederman, 2002). The discrimination of these "five modes of touch" refers to perception after exploring a real object. Considering tactile and haptic devices as a way of perceiving an object and its properties, we expanded these five modes of touch adding a sixth mode of touch as a subcategory of the tactile (cutaneous) perception which is the "tactile stimulus motion perception". According to the criterion of presence or absence of motor control this mode is passive due to the fact that there is no human motion but there is a stimulus motion. A stimulus motion can be provoked either by moving the signal applied to the skin (created by appropriate actuators located on a device) or by moving the object beneath the human finger or hand. The final stage of processing of a haptic system is the extraction of the attributes of surfaces and objects. Combined perception of distinct attributes of touched objects leads to the recognition of objects. The sensory perception class has two subclasses according to the haptically perceptible properties of touched objects: Perception of geometry properties and Perception of material properties. The Perception of geometry properties class has four subclasses that represent the haptically perceptible properties of touched objects from the geometry aspect and they are the following: Perception of size, Perception of shape, Perception of curvature and Perception of weight. Weight is a property which reflects geometry and material. Although an object's weight is defined by its total mass, perceived weight can be affected by the object's material, shape, and identity (Klatzky \& Lederman, 2002). The Perception of material properties class has four subclasses which are the following: Perception of hardness, Perception of roughness, Perception of temperature and Perception of texture.

The second subclass of the span:Processes class is the stimuli class. The stimuli class includes the different kind of stimuli that the human skin can receive and can activate the receptors. The stimuli class is divided into the following subclasses according to the stimuli types: chemical stimuli, electrical stimuli, mechanical stimuli and thermal stimuli. Stimulus features are processed in parallel by distinct areas of the brain cortex.

- Aggregates of processes: They are moreological sum of processes (Grenon et al., 2004). Subclass of the span:Aggregates of processes class is the touch modality class. Touch modality is the "channel" originating in the tactile device that uses the skin to communicate with the receptors and the nerves to transmit signals to the brain. Touch modality is determined by the type of energy transmitted by the different kind of stimuli to the skin using specific mechanical, electrical, thermal properties of the skin, the receptors specialized to sense that energy, the neural activity generated by the receptors and the brain areas which accept the neural activity which leads to perception of specific tactile features. Touch modality is a complex process derived from the combination of different kind of stimuli and different energy types that are transmitted to the receptors. Touch modality class 
includes the following subclasses in agreement with the different submodalities of touch modality: Pressure, Skin stretch, Stroking, Fluttering, Vibration, Coldness, Warmth, Pain, Muscle tension, Muscle stretch and Joint tension.

- Fiat process part: It is a processual entity that is part of a process but does not have beginnings or endings corresponding to real discontinuities. Tactile signal transmission by receptors, tactile signal transmission by nerves and tactile signal processing by brain areas are subclasses of span:FiatProcessPart class as parts of tactile information process.

- Process boundaries: They are processual entities or events that are instantaneous temporal boundaries of processes. In this class of occurrents belongs the user interaction with a tactile device.

- Patterns of change: Time and change are intrinsically represented within every single instance of a SPAN ontology in the form of morphological transformations and separations of the temporally extended processes that are represented. SPAN captures the flowing and continuous nature of processes by representing change in terms of a constant and connected succession of temporal parts or stages. Nevertheless, it is in principle always possible to choose any two instants of time in a SPAN ontology, and to compare the process parts existing at those instants of time in order to determine if they are identical, or if change has occurred (Spear, 2006).

Tactile information is generated whenever a change in the attributes of processes and especially the stimuli processes occurs. In order to define and describe the generation of tactile information we have to describe the patterns of change of: the location of the touch modality, the modality range, the touch modality time range and the magnitude of the touch modality.

The hierarchy of processual entities is presented in Fig. 9.

\subsubsection{Temporal regions}

They are the regions (intervals) of time during which SNAP and SPAN entities exist. Temporal regions are divided into: connected temporal regions, every point of which is connected to every other point, and scattered temporal regions, every point of which is not mediately or immediately connected to every other point. The span:ConnectedTemporalRegion class has two subclasses: span:TemporalInstant and span:TemporalInterval. Temporal instants are connected temporal regions comprising a single moment of time. In tactile information ontology, a subclass of span:TemporalInstant is Receptors activation comprising the instance of time that a stimulation occurs. The span:TemporalInterval class includes connected temporal regions lasting for more than a single moment of time. In tactile information ontology, the subclasses of span:TemporalInterval are: brain processing duration comprising the interval of time that tactile signals are processed in the brain regions, cortical neurons activation comprising the time duration of cortical neurons activation, nerves transmission time defining the duration of tactile signals transmission through nerves and receptors stimulation comprising the time duration of a stimulus that activates a receptor (Fig. 10).

\subsubsection{Spatiotemporal regions}

Spatiotemporal regions are occurrents at or in which processual entities can be located. The totality of spatiotemporal regions reflects the totality of possible demarcations of that maximal region we call space-time. Processuals stand to spatiotemporal regions in a way analogous to the way in which substantials stand to spatial regions. In our case, they are regions of space-time which are parts of the spatiotemporal location of the human-tactile system interaction process. Due to the fact that spatiotemporal region's importance is primarily theoretical and it is not used often in most domain ontology applications, we did not expand this class to subclasses for the tactile information domain. 


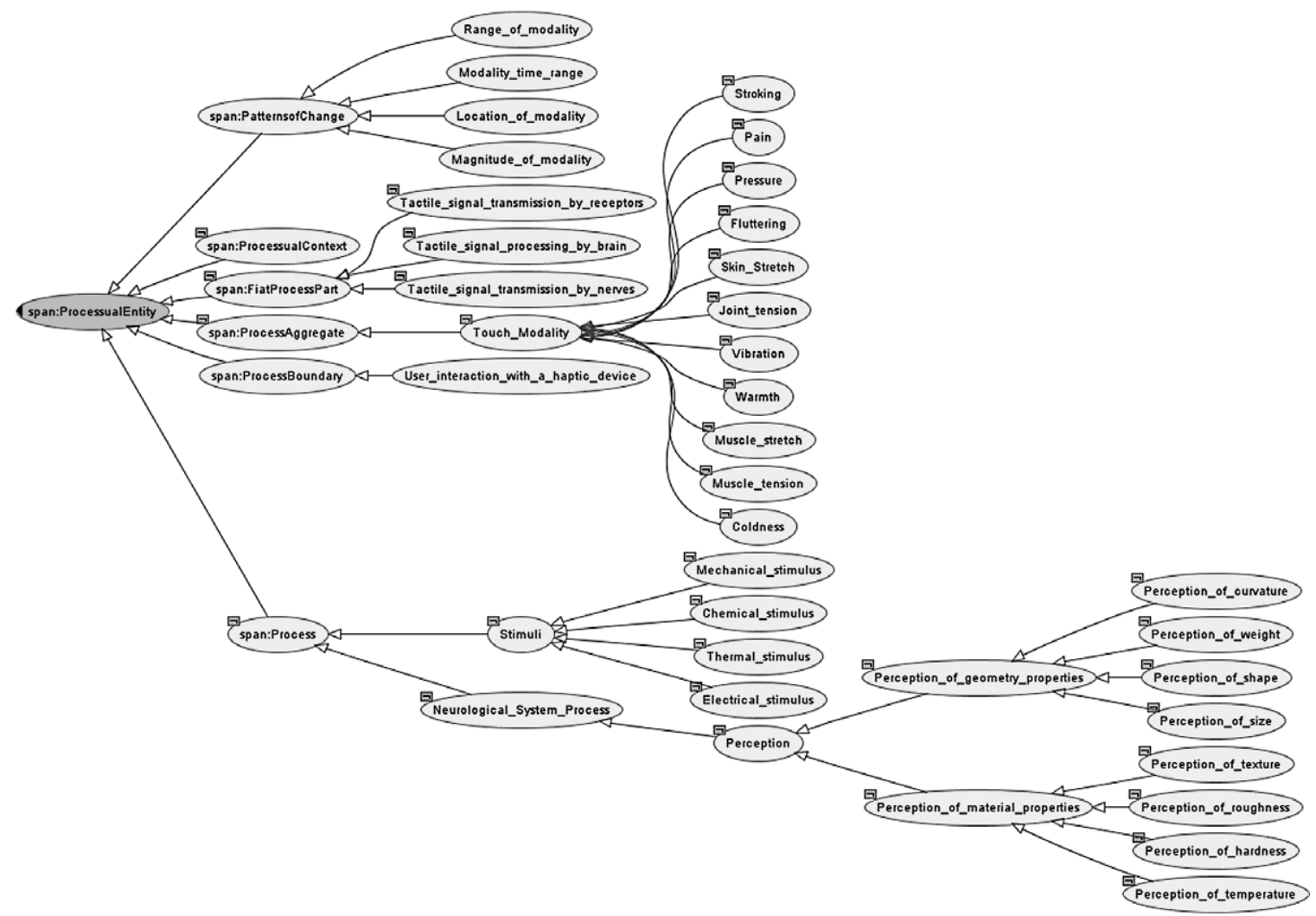

Fig. 9. The processual entities hierarchy.

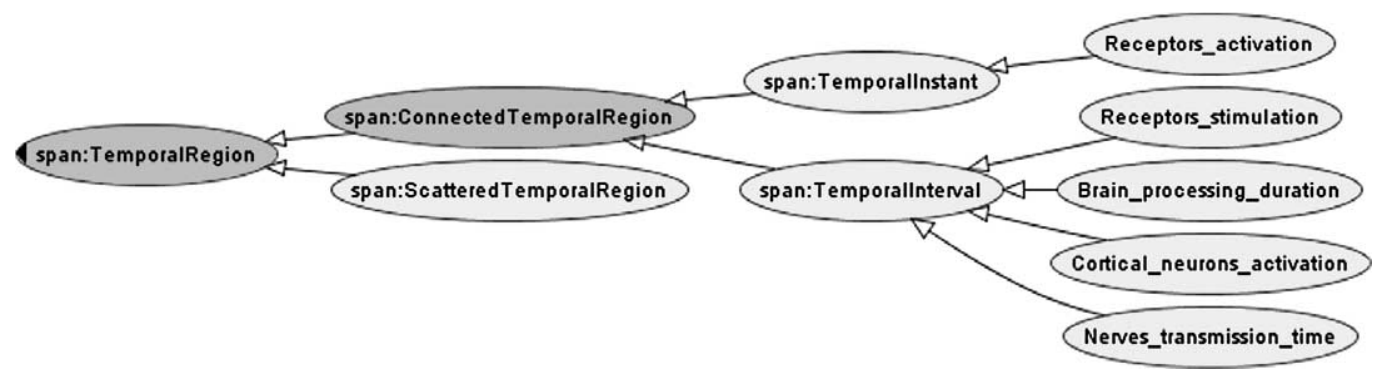

Fig. 10. The temporal regions hierarchy.

\section{Ontological relations}

As has already been noted in previous sections, specification of entities and ontological categories alone are not enough to adequately capture all of the important information about a given domain. Rather, the relationships obtained amongst the classes and categories in an ontology need to be represented (Spear, 2006). When adding a relationship to any ontology, whether formal or domain specific, it is crucial that it should be clear what kind of entities these relationships hold.

There are two types of relations in BFO: intra-ontological and trans-ontological (Grenon et al., 2004). Intra-ontological relations are relations between entities all of which are constituents of a single ontol- 


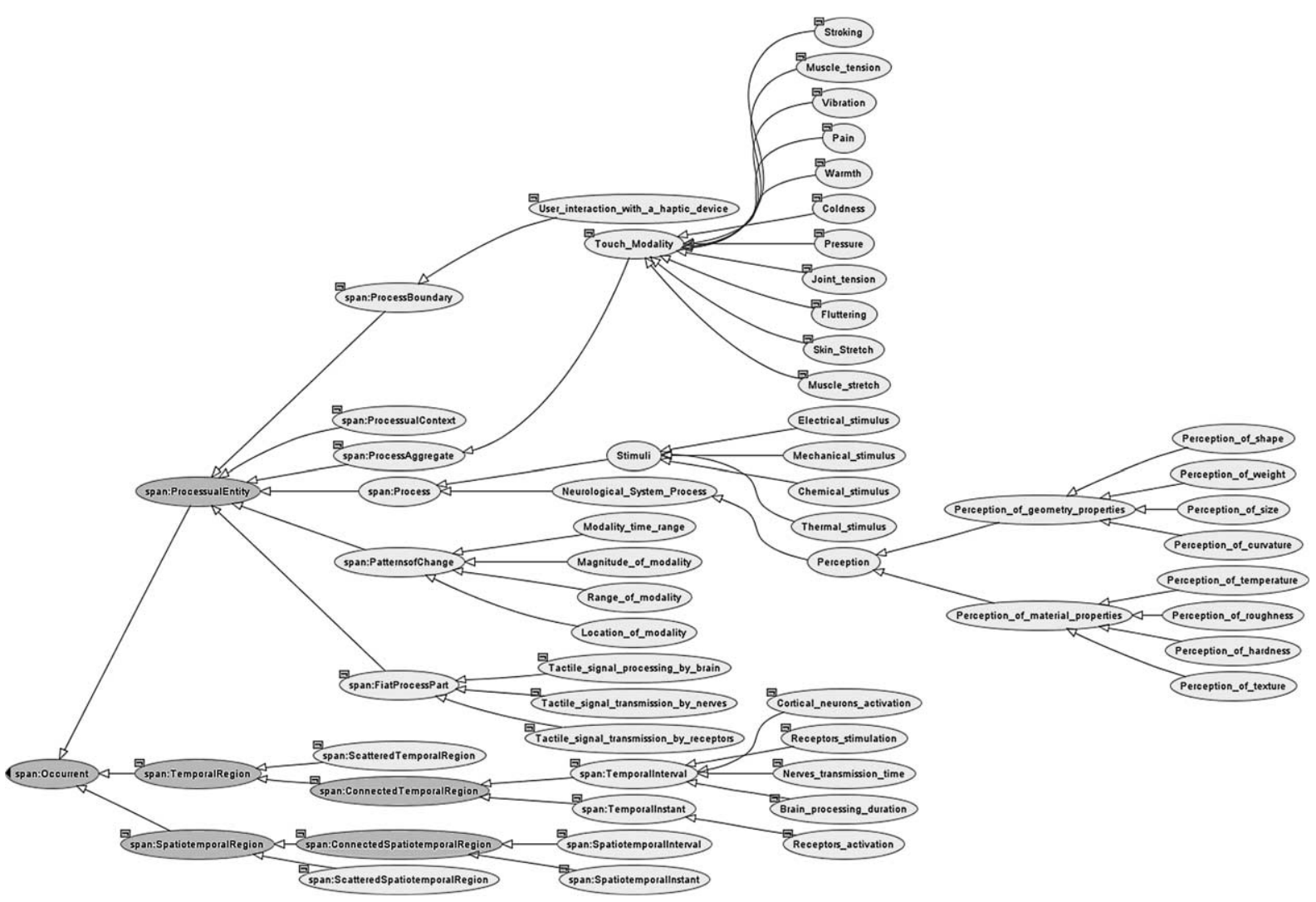

Fig. 11. The SPAN tactile information hierarchy.

ogy. There is a discrimination between the two main types of intra-ontological relations reflecting the SNAP-SPAN opposition. Part-whole and topological relations are always intra-ontological, and thus they never cross the SNAP-SPAN divide: an object is never a part of a process and a process is never a part of an object. The two fundamental intra-ontological relationships are the "is-a" relation and "partof" relation (Grenon et al., 2004; Grenon \& Smith, 2004; Smith et al., 2003). Trans-ontological are relations between entities that are constituents of distinct ontologies, such as the relation of participation between an object and a process (as when the fingers of a hand participate in a tactile device operation).

Examples of relations between SNAP and SPAN entities include: dependence, participation, creation, initiation, termination and destruction.

\subsection{The "is-a" relation}

The "is- $a$ " relation is a relation that is clearly used in a way to indicate is a kind of specialized relations between classes. The "is- $a$ " relation indicates a necessary relation. That is, when we say "mechanoreceptor is a receptor", we mean that every mechanoreceptor is a receptor. In SNAP tactile information ontology, receptors class and its subclasses are connected with "is- $a$ " relations and nerves class and its subclasses are connected with "is-a" relations. Furthermore, geometry quality class and its subclasses, material quality class and its subclasses are also connected with "is- $a$ " relations. 
In SPAN tactile information ontology, neurological system process "is-a" process, perception "is- $a$ " neurological system process. Each stimulus (mechanical, electrical, thermal, chemical) subclass "is-a" stimulus, each touch modality (pressure, skin stretch, stroking, warmth, coldness, pain, vibration) "is-a" modality. Moreover, nerves transmission time "is-a" temporal interval, cortical neurons activation "is-a" temporal interval, receptors stimulation "is-a" temporal interval, brain process duration " $i s-a$ " temporal interval and receptors activation "is- $a$ " temporal instant.

\subsection{The "part-of" relation}

The "part-of" relation (also referred as parthood relation or temporary parthood relation when it is referred to processes) (Masolo et al., 2003) is a relation between instances (Bittner et al., 2004). Tactile information ontology uses "part-of" for representing parts of both substances and processes and of functions/activities. In SNAP tactile information ontology, it appears in the following:

- palm and fingertip are "part-of" the skin surface,

- nerves, brain, joints, muscles, tendons are "part-of" the human body,

- brain cortex is "part-of" the brain,

- primary somatic cortex, secondary somatic cortex and posterior cortex are "part-of" the brain cortex,

- area 1, area 2, area 3a, area 3b are "part-of" primary somatic cortex,

- area 5, area 7 are "part-of" posterior cortex.

In SPAN tactile information ontology the parthood relation appears between the following classes:

- perception of geometry properties is "part-of" the sensory perception,

- perception of material properties is "part-of" the sensory perception,

- tactile signal transmission by the receptors is "part-of" the tactile information process,

- tactile signal transmission by the nerves is "part-of" the tactile information process,

- tactile signal processing by the brain is "part-of" the tactile information process.

\subsection{Relations between SNAP and SPAN entities}

For purposes of ontology construction the primary relationships we are interested in are those obtained between classes, including but not limited to the "is-a" or "part-of" relation. However, it is important to address and define relationships obtained between instances and also relationships obtained between classes and instances because these may be sometimes important to represent an ontology, and also because definitions of such relations can themselves help to play a role in defining the explicit relations obtained between classes (Spear, 2006). In this section we are going to describe the trans-ontological relation between SNAP and SPAN entities and classes.

\subsubsection{Dependence}

Dependence or inherence is a relation that connects dependent entities (such as roles, functions, qualities) with objects or processes. Dependence of an entity $x$ on an entity $y$ is defined as follows: $x$ depends on $y$ if presence of $y$ necessarily implies presence of $x$. In our case, this kind of relations exist in:

Functions: the cortical neurons function that is dependent on the brain or inheres in the brain, the function of a haptic device that is dependent on the haptic device used, the neural canals function that is dependent on the nerve canals and the function of receptors activation that is dependent on the receptors. 
Roles: the roles of the brain, nerves and receptors in tactile information process are dependent on the brain, nerves and receptors respectively.

Qualities: Quality entities that in our case represent the haptically perceptible properties of an object that can be touched through a haptic device, inhere or depend on the object that a user can manipulate through a haptic device (Klatzky \& Lederman, 2002).

\subsubsection{Participation}

Participation is a relation that represents how continuants are "involved" in an occurrent. Participation should not be confused with parthood relation because the continuants can participate in occurrents but are not parts of occurrents. In tactile information ontology, brain participates in tactile signal processing, proprioception and tactile perception processes. A haptic device is an object that participates in the human-haptic system interaction process and also participates in the stimulation process creating stimuli. Skin receptors participate in tactile signal transmission to the nerves and the nerves participate in tactile signal transmission to the brain while material and geometry object properties participate in perception.

\subsubsection{Physical location}

The relation "located in" connects an entity with another entity that hosts its location. Proprioceptors are located in joints and muscles, mechanoreceptors are located in skin layers, palm and fingertip are located in skin surface, brain is located in the cranial cavity, nerves are located in nerve channels, brain areas are located in primary and somatic cortices.

\subsubsection{Spatiotemporal location}

As we have mentioned above, spatial regions are related to substantial entities and spatiotemporal and temporal regions are related to processual entities. Substantial entities are related to spatial regions with the relation "spatially related to" and processes are related to temporal regions with the relation "temporally related to".

\subsection{Tactile interaction relations}

The ontology presented in this work includes classes and relations that represent the objects and the processes involved in the human-tactile system interaction. Tactile information process consists of a series of events that include different kind of entities. Apart from the formal ontological relations between classes that have been described above, we have analyzed the relations involved in the various steps of the human-tactile system interaction process, presented in Fig. 12. The relations between objects and processes that take part in the tactile information generation and processing are the following: a human "interacts with" a haptic system. Haptic/tactile device "receives" tactile features of a touched object through the device actuators causing direct actuator stimulation. The haptic device class is connected to the touch modality class with the relation "mediates sensation" due to the fact that haptic devices mediates sensations such as pressure, skin stretch, vibration, warmth, coldness, joint tension, muscle tension and pain. Tactile information is "generated by" the changes of the touch modalities properties (location, time range, magnitude) that are defined in the Patterns Of Change class.

The haptic device class is connected to the stimuli class with the relation "produces". The haptic/tactile device "transmits" a stimulus (mechanical, chemical, electrical, thermal) to the receptors. Receptors "receive" stimuli from the device and after processing, they "transmit" tactile signals to the nerves. Nerves "transmit" tactile signals to the brain cortex through different pathways while the brain cortex "distributes" these signals to brain areas. Brain areas are responsible to "integrate" perception. 


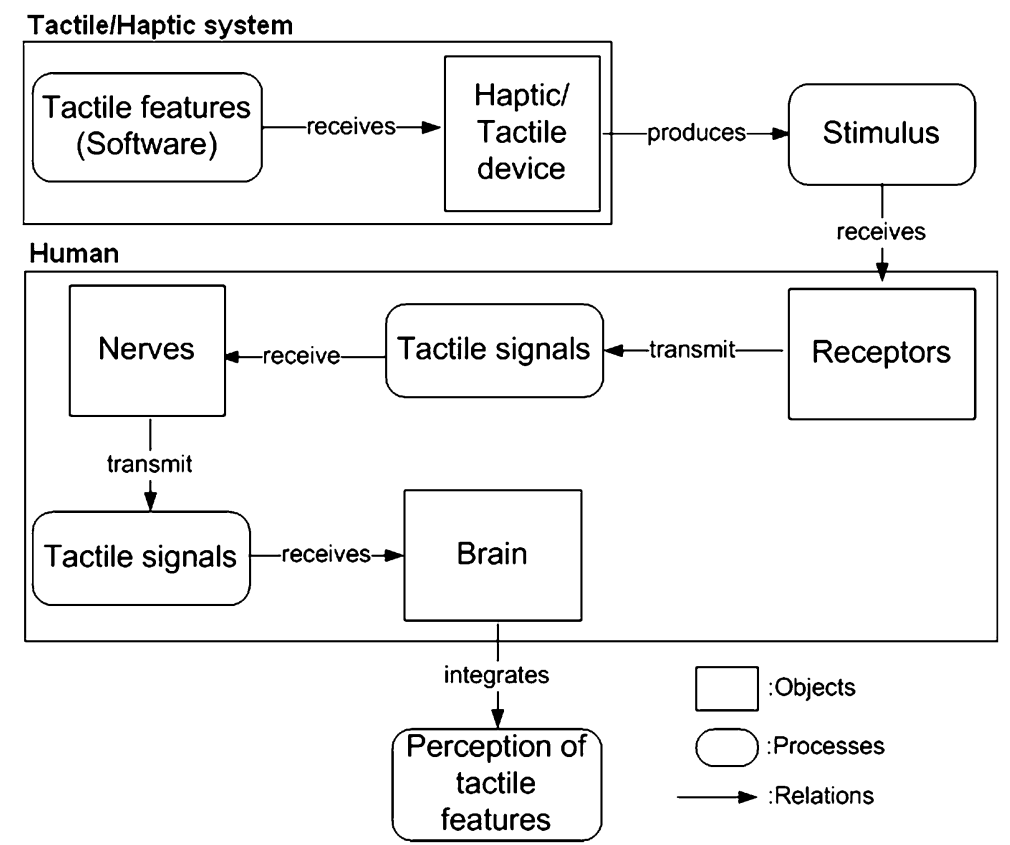

Fig. 12. Relations corresponding to different steps in information flow in tactile information ontology.

\subsubsection{Relation between touch modality classes and receptors classes}

Each kind of receptors is activated by a different touch modality. $R A-I$ mechanoreceptors are "activated by" stroking and fluttering, RA-II mechanoreceptors are "activated by" vibration, SA-I class is "activated by" local stress-strain (pressure) and SA-II is "activated by" skin stretch. Golgi endings are "activated by" joint tension, Golgi tendons are "activated by" muscle tension and Muscle spindles are "activated by" muscle stretch. Cold receptors are "activated by" coldness touch modality and warm receptors are "activated by" warmth touch modality. Nociceptors are "activated by" pain. The changes in touch modality properties that are defined in the Patterns of change class "generate" tactile information that is "conveyed to" the receptors through the modalities.

\subsubsection{Relation between receptors classes and nerves classes}

The result of processing in the receptors is the generation of tactile signals that are transmitted through the nerve fibers in separate pathways. The cells that make up these pathways have distinctive response properties inasmuch as each pathway conveys information from a different kind of receptor. For each receptor kind, tactile signals are transmitted through different kinds of nerves. Receptors class and nerves class are connected with the relation "connected through". In this relation the first participant transmits and the second receives. Mechanoreceptors class is "connected through" A $\beta$ fibers, cold thermoreceptors and nociceptors are "connected through" A $\delta$ fibers, warm thermoreceptors and nociceptors are "connected through" C fibers, golgi endings and golgi tendons are "connected through" Ib fibers and muscle spindles are "connected through" Ia and II fibers.

\subsubsection{Relation between receptors classes and brain areas classes}

Each area of the brain that is located in the brain cortex is responsible to receive signals from different classes of receptors. Area 1 "receives" input from rapidly adapting receptors and area $3 b$ "receives" signals from mechanoreceptors. Specifically, area $3 \mathrm{~b}$ computes differences in activity of adjacent SA-I 
receptors which are passed along to S-II (Klatzky \& Lederman, 2002). Area 3 a "receives" input signals from muscle spindles and area 5 "receives" inputs signals from proprioceptors, while area 2 "receives" input from cutaneous and deep receptors. Secondary somatic cortex "receives" input from the primary somatic cortex.

\subsubsection{Relation between brain areas classes and perception classes}

The role of brain areas in human-tactile system interaction is the integration of perception and the extraction of the touched object's properties. To describe this event we created the relation "integrates". Specifically, area $3 b$ "integrates" perception of object's roughness, hardness and texture, area 1 "integrates" perception of object's size and texture, area 2 "integrates" perception of object's curvature, area 5 "integrates" perception of object's shape and finally, area 7 contributes to perception of size and shape.

\section{Case study}

In this section we describe a case study (Fig. 13) based on our ontology, relative to the perception of roughness generated by a suitable tactile device. Roughness characterizes the texture of a surface using the roughness measure and is quantified by the vertical deviation of a real surface from its ideal form (smooth). If these deviations are large, the surface is rough; if they are small the surface is smooth. ${ }^{9}$ In our case study, a data flow diagram is used presenting the sequence of actions performed by a tactile system and a human system and how the final output (perception) is produced.

Our case study includes two systems: the tactile and the human system. The output data of the tactile system is the input to the human system. A tactile system consists of hardware and software that drives the device while the human system consists of the "channel" (receptors, nerves, brain). The tactile device we consider for our case study is a tactile display which creates a stress field along the finger using acoustic radiation pressure. The tactile system consists of a linear array of PZT transducers and a driving circuit. It uses phased array technology for beam steering and focusing. The result is the production of various precise spatiotemporal patterns of pressure distribution on the skin (Iwamoto \& Shinoda, 2005).

In general, tactile software uses a geometry model of the surface that the human will perceive through the display and from that it extracts the tactile features. The spatial distribution of these features is used to create a mathematical model of stimulus. This model is the input to the tactile hardware that consists of the device controller and the device. The device controller processes the stimulus model and generates a signal that drives the device producing the actual stimulus that is transmitted to the human.

We considered a rough surface and in order to simplify our model, we considered a line on this surface along $x$ axis. The data flow diagram (Fig. 13) describes the following sequence processes: using the geometry model that represents the surface we extract the measure of roughness $R(x)$. The mathematical model of stimulus that represents the pressure field in relation to roughness measure, is defined as $P^{\prime}(x)$. This stimulus model is the input to the device controller which produces a voltage signal $V(x, \varphi)$, where $\varphi$ is the pulse phase and the device hardware converts it to sound intensity signal $I(x, \varphi)$. This intensity signal through air and interference creates the pressure stimulus $P(x)$.

The pressure stimulus is the input to the human system. Specifically, pressure stimulates different points of the skin and activates the mechanoreceptors, and especially SA-I (Merkel cells), which create

\footnotetext{
${ }^{9}$ http://en.wikipedia.org/wiki/Roughness.
} 


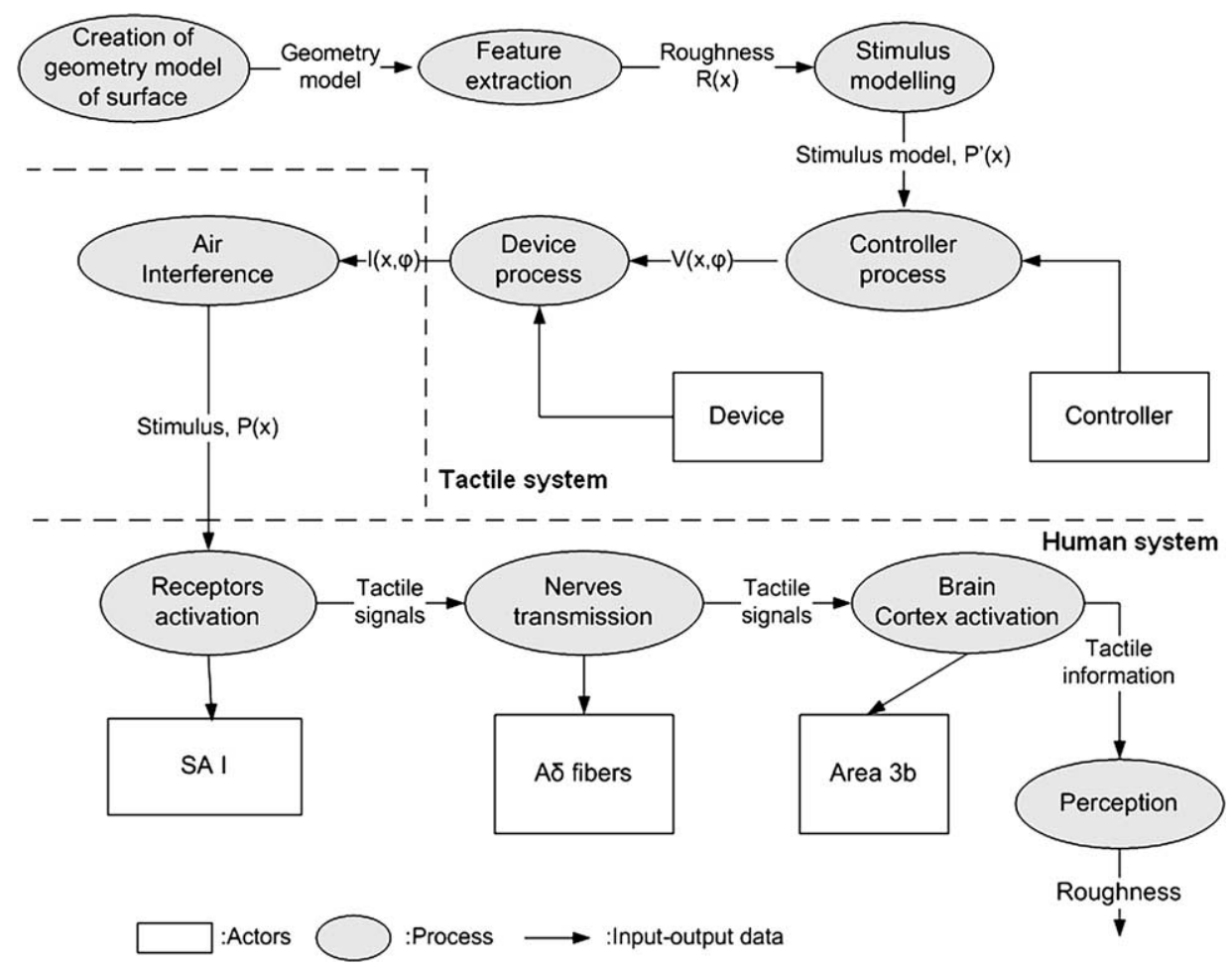

Fig. 13. Data flow diagram.

neural activity that is transmitted as tactile signals through the nerves. The nerve fibers that are responsible to receive and transmit the neural activity from SA-I receptors are A $\beta$ fibers, which finally drive the primary somatic cortex and especially the area $3 \mathrm{~b}$. It is known that this area is responsible for the perception of roughness based on the computation of differences in activity of adjacent SA-I receptors.

\section{Summary and future work}

In this paper we have described the development of a tactile information ontology, in relation to the entities that take part in the tactile information flow. The ontology design is based on the generation of tactile information by a tactile device, the transmission and processing of these signals by the skin receptors, the nerves and the brain, and the integration of perception. Perception is the result of the human-tactile system interaction and due to this fact we needed to extend the existing modes of touch perception adding a new mode of perception that can be achieved using a tactile or haptic device. This additional mode will help us in better understanding of human-tactile system interaction and modelling of the software for tactile devices. The ontological representation of tactile information provides a source of precisely defined terms and relations that can be communicated across people and software tools. However, the designed ontology is not an ontology describing the sense of touch whereas it is an ontology that models the sense of tactation in order to implement human-computer interaction with tactile and haptic devices.

The ontology structure follows the Basic Formal Ontology (BFO), which is an upper-level ontology that can be used in support of domain ontologies. Ontology development has been done with the Pro- 
tege OWL Editor which provides to the growing software user community a suite of tools to construct domain models and knowledge-based applications, and enables the exportation of ontologies in many different formats. The proposed model was developed to address two open issues. First, we formalized the vocabulary that describes human-tactile system interaction, providing a formal definition of tactile information that can be utilized by users and applications. Second, the ontology we have developed for the tactile information domain will hopefully help in designing better software for tactile interfaces. Moreover, we believe that the proposed ontology could serve as a framework to allow an easy acquisition of knowledge about tactile information using tactile and haptic devices.

The ontology proposed allows us to specify the above notions with great clarity since all the entities, objects and processes, that take part in the human-tactile system interaction are considered. The following concepts are explicitly described: the different kind of stimuli produced by a haptic device and transmitted to the receptors, the tactile signals generated by stimulating the skin receptors, the transmission of these signals through the nerves and the processing of received information by the brain that finally leads to perception of the tactile features of a touched object. Moreover, the relations between entities have been represented and analyzed in detail in order to build a complete model and describe adequately the tactile information domain. Specifically, formal relations introduced in upper level ontologies as well as relations belonging to the tactile information domain have been considered. The resulting ontology will help us in better understanding of tactile sensation because it defines all possible ways of sensation through touch, and perception of information that is created by a tactile device. It is important to emphasize that this approach can be used to define tactile information classes in object-oriented language and to assist software development.

A case study has been also developed in order to validate our ontology. Considering the perception of roughness, the case study involve an in-depth, longitudinal examination of this event. It represents the processes and the data that are produced by a tactile software system, a tactile hardware system and the human system. The case study that we described is a data flow diagram and includes the data and events that take part in human-tactile system interaction and will support the design of software for tactile devices.

Further research is directed to the construction of an object-oriented model according to this ontological representation, and formally represent information about the tangible features of objects (size, shape, texture, etc.) which is our main purpose. The addition of an object-oriented model to this ontology will provide additional levels of details not found in this ontology (class, object, methods, inheritance, composition, encapsulation and polymorphism concepts), increasing the depth of information that can be applied to the description of a tactile process. This class hierarchy can be translated directly into code, facilitating the creation of simulations and providing the tools to tactile device software development.

\section{Acknowledgements}

This paper is part of the 03ED375 research project, implemented within the framework of the "Reinforcement Programme of Human Research Manpower" (PENED) and co-financed by National and Community Funds (25\% from the Greek Ministry of Development - General Secretariat of Research and Technology and 75\% from E.U. - European Social Fund). 


\section{References}

Bittner, T. \& Barry, S. (2004). Normalizing medical ontologies using basic formal ontology. In Proceedings of GMDS (pp. 199-201).

Bittner, T., Donnelly, M. \& Smith, B. (2004). Individuals, universals, collections: On the foundational relations of ontology. Technical report, University of Leipzig, Germany.

Bohlhalter, S., Fretz, C. \& Weder, B. (2002). Hierarchical versus parallel processing in tactile object recognition. Brain, 125, $2537-2548$.

Bolanowski, S.J., Gescheider, G.A., Verillo, R.T. \& Checkosky, C.M. (1988). Four channels mediate the mechanical aspects of touch. Journal of Acoustical society, 84(5), 1680-1694.

Bonacin, R., Baranauskas, C.C. \& Liu, K. (2004). From ontology charts to class diagrams: semantic analysis aiding systems design. In Proceedings of the 6th International Conference on Enterprise Information Systems (Vol. 3, pp. 389-395).

Chandrasekaran, B., Josephson, J.R. \& Benjamins, R.V. (1999). What are ontologies and why do we need them? IEEE Intelligent Systems, 14(1), 20-26.

Cranefield, S. \& Purvis, M. (1999). Uml as an ontology modeling language. In Proceedings of the Workshop on Intelligent Information Integration, 16th International Joint Conference on AI (IJCAI-99) (pp. 46-53).

Darian-Smith, I. \& Johnson, K.O. (1977). Thermal sensibility and thermoreceptors. The Journal of Investigative Dermatology, 69, 146-153.

Devedzic, V. (2002). Understanding ontological engineering. Communications of the ACM, 45(4), 136-144.

Evermann, J. \& Wand, Y. (2005). Ontology based object-oriented domain modelling: fundamental concepts. Requirements Engineering, 10, 146-160.

Gangemi, A., Guarino, N., Masolo, C., Oltramari, A. \& Schneider, L. (2002). Sweetening ontologies with dolce. In Proceedings of EKAW (pp. 166-181).

Gardner, E. \& Kandel, E. (1991). Principles of Neural Science, 4th edn (Chapter “Touch”, pp. 452-472). Elsevier.

Gardner, E. \& Martin, J. (1991). Principles of Neural Science, 4th edn (Chapter “Coding of sensory information”, pp. 412-430). Elsevier.

Gardner, E., Martin, J. \& Jessel, T. (1991). Principles of Neural Science, 4th edn (Chapter “The bodily senses”, pp. 431-451). Elsevier.

Gescheider, G.A., Bolanowski, S.J. \& Verrillo, R.T. (2004). Some characteristics of tactile channels. Behavioural Brain Research, 148, 35-40.

Gescheider, G.A., Thorpe, J.M., Goodarz, J. \& Bolanowski, S.J. (1997). The effects of skin temperature on the detection and discrimination of tactile stimulation. Somatosensory \& Motor Research, 14(3), 181-188.

Gomez-Perez, A. (2003). Ontological engineering: A state of the art. Expert Update, 2(3), 33-42.

Grenon, P. (2003). Spatio-temporality in basic formal ontology, part i: Snap and span, upper-level ontology and framework for formalization. Technical report, IFOMIS Group.

Grenon, P. \& Smith, B. (2004). Snap and span: Towards dynamic spatial ontology. Spatial Cognition and Computation, 4(1), 69-104.

Grenon, P., Smith, B. \& Goldberg, L. (2004). Ontologies in Medicine (Chapter "Biodynamic ontology: Applying BFO in the biomedical domain", pp. 20-38). IOS Press.

Gruber, T.R. (1993). Toward principles for the design of ontologies used for knowledge sharing. International Journal HumanComputer Studies, 43, 907-928.

Hale, K.S. \& Stanney, K.M. (2004). Deriving haptic design guidelines from human physiological, psychophysical and neurological foundations. IEEE Computer Graphics and Applications, 24(2), 33-39.

Hlushchuk, Y. \& Hari, R. (2006). Transient suppression of ipsilateral primary somatosensory cortex during tactile finger stimulation. The Journal of Neuroscience 26(21), 5819-5824.

Hruby, P. (2005). Role of domain ontologies in software factories. In OOPSLA 2005 Workshop on Software Factories. San Diego, CA. Available at: http://softwarefactories.com/workshops/OOPSLA-2005/Papers/Hruby.pdf.

Iggo, A. (1984). Cutaneous receptors and their sensory functions. The Journal of Hand Surgery, 9(1), 7-10.

Iwamoto, T. \& Shinoda, H. (2005). Ultrasound tactile display for stress field reproduction - examination of non-vibratory tactile apparent movement. In First Joint Eurohaptics Conference and Symposium on Haptic Interfaces for Virtual Environment and Teleoperator Systems (WHC'05) (pp. 220-228).

Johansson, R.S. (1978). Tactile sensibility in the human hand: Receptive field characteristics of mechanoreceptive units in the glabrous skin area. Journal of Physiology, 281, 101-123.

Johnson, K.O. (2001). The roles and functions of cutaneous mechanoreceptors. Current Opinion in Neurobiology, 11, 455-461.

Khan, L. \& Luo, F. (2002). Ontology construction for information selection. In Proceedings of the 14th IEEE International Conference on Tools with Artificial Intelligence (ICTAI'02) (pp. 122-127). 
Klatzky, R.L. \& Lederman, S.J. (2002). Experimental Psychology (Vol. 4, Chapter “Touch”, pp. 147-176). Wiley.

Kogut, P.A., Cranefield, S., Hart, L., Dutra, M., Baclawski, K., Kokar, M.M. \& Smith, J.E. (2002). Uml for ontology development. The Knowledge Engineering Review, 17(1), 61-64.

Loomis, J.M. \& Lederman, S.J. (1986). Handbook of Perception and Human Performance (Vol. II, Chapter “Tactual perception").

Macefield, V. (2005). Physiological characteristics of low-threshold mechanoreceptors in joints, muscle and skin in human subjects. Clinical and Experimental Pharmacology and Physiology, 32, 135-144.

Masolo, C., Borgo, S., Gangemi, A., Guarino, N., Oltramari, A. \& Schneider, L. (2003). The wonderweb library of foundational ontologies. Technical report, ISTC-CNR.

Masolo, C., Vieu, L., Bottazzi, E., Catenacci, C., Ferrario, R., Gangemi, A. \& Guarino, N. (2004). Social roles and their descriptions. In D. Dubois, C. Welty \& M. Williams (eds), Ninth International Conference on the Principles of Knowledge Representation and Reasoning (KR2004) (pp. 267-277).

Mika, P. (2007). Social Networks and the Semantic Web. Springer.

Myrgioti, E., Chouvardas, V., Miliou, A. \& Hatalis, M. (2007). Modeling tactile information flow using ontologies. In 3rd Balkan Conference in Informatics (BCI 07) (Vol. 2, pp. 219-228).

Niles, I. \& Pease, A. (2001). Towards a standard upper ontology. In C. Welty \& B. Smith (eds), 2nd International Conference on Formal Ontology in Information Systems (FOIS-2001) (pp. 2-9).

Noy, N., Sintek, M., Decker, S., Crubezy, M., Fergerson, R. \& Musen, M. (2001). Creating semantic web contents with protege2000. IEEE Intelligent Systems, 16(2), 60-71.

Noy, N.F. \& Hafner, C. (1997). The state of the art in ontology design. American Association for Artificial Intelligence (pp. 5374).

Noy, N.F. \& McGuiness, D. (2001). Ontology Development 101: A guide to creating your first ontology. Knowledge Systems Laboratory.

Pasterkamp, E. (1999). Mechanoreceptors in the glabrous skin of the human hand. Archives of Physiology and Biochemistry, 107(4), 338-341.

Pearson, K. \& Gordon, J. (1991). Principles of Neural Science, 4th edn (Chapter "Spinal reflexes", pp. 714-737). Elsevier.

Recanzone, G.H., Merzenich, M.M. \& Schreiner, C.E. (1992). Changes in the distributed temporal response properties of si cortical neurons reflect improvements in performance on a temporally based tactile discrimination task. Journal of Neurophysiology, 67(5), 1071-1091.

Rubin, D.L., Shah, N.H. \& Noy, N.F. (2007). Biomedical ontologies: a functional perspective. Briefings in Bioinformatics, 9(1), 75-90.

Ruiz, F. \& Hilera, J. (1998). Ontologies for Software Engineering and Software Technology (Chapter "Using ontologies in software engineering and technology”, pp. 49-102). Springer.

Shegogue, D. \& Zheng, W.J. (2005). Integration of the gene ontology into an object-oriented architecture. BMC Bioinformatics, 6(113), 113-127.

Smith, B., Williams, J. \& Steffen, S. (2003). The ontology of the gene ontology. In AMIA Symposium 2003 (pp. 609-613).

Spear, A.D. (2006). Ontology for the twenty first century: An introduction with recommendations (pp. 1-132). Internal report, Institute for Formal Ontology and Medical Information Science (IFOMIS), Saarbrucken, Germany University at Buffalo, Buffalo, New York.

Uschold, M. \& Gruninger, M. (1996). Ontologies: Principles, methods and applications. Knowledge Engineering Review, 11(2), 93-136.

Uschold, M. \& King, M. (1995). Towards a methodology for building ontologies. In Workshop on Basic Ontological Issues in Knowledge Sharing, held in conjunction with the Fourteenth International Joint Conference on Artificial Intelligence (IJCAI'95), Montreal, Quebec. (Also available as AIAI-TR-183 from AIAI, The University of Edinburgh.)

Vallbo, A.B., Hagbarth, K.E., Torebjork, H.E. \& Wallin, B.G. (1979). Somatosensory, proprioceptive, and sympathetic activity in human peripheral nerves. Physiological Reviews, 59(4), 919-957.

van Heijst, G., Schreiber, A.T. \& Wielinga, B.J. (1997). Using explicit ontologies in kbs development. International Journal of Human-Computer Studies, 45, 183-292.

Verrillo, R.T. (1985). Psychophysics of vibrotactile stimulation. Acoustical Society of America, 77(1), 225-232. 